NBER WORKING PAPER SERIES

\title{
CAPITAL DEEPENING AND NON-BALANCED ECONOMIC GROWTH
}

\author{
Daron Acemoglu \\ Veronica Guerrieri \\ Working Paper 12475 \\ http://www.nber.org/papers/w12475
NATIONAL BUREAU OF ECONOMIC RESEARCH
1050 Massachusetts Avenue
Cambridge, MA 02138
August 2006

We thank John Laitner, Guido Lorenzoni, Iv an Werning and seminar participants at Chicago, Federal Reserve Bank of Richmond, IZA, MIT, NBER Economic Growth Group, 2005, Society of Economic Dynamics, Florence 2004 and Vancouver 2006, and Universitat of Pompeu Fabra for useful comments and Ariel Burstein for help with the simulations. Acemoglu acknowledges financial support from the Russell Sage Foundation and the NSF. An early version of this paper was circulated under the title "Non-Balanced Endogenous Growth". The views expressed herein are those of the author(s) and do not necessarily reflect the views of the National Bureau of Economic Research.

(O2006 by Daron Acemoglu and Veronica Guerrieri. All rights reserved. Short sections of text, not to exceed two paragraphs, may be quoted without explicit permission provided that full credit, including $\odot$ notice, is given to the source. 
Capital Deepening and Non-Balanced Economic Growth

Daron Acemoglu and Veronica Guerrieri

NBER Working Paper No. 12475

August 2006

JEL No. O30, O40, 041

\begin{abstract}
$\underline{\text { ABSTRACT }}$
This paper constructs a model of non-balanced economic growth. The main economic force is the combination of differences in factor proportions and capital deepening. Capital deepening tends to increase the relative output of the sector with a greater capital share, but simultaneously induces a reallocation of capital and labor away from that sector. We first illustrate this force using a general two-sector model. We then investigate it further using a class of models with constant elasticity of substitution between two sectors and Cobb-Douglas production functions in each sector. In this class of models, non-balanced growth is shown to be consistent with an asymptotic equilibrium with constant interest rate and capital share in national income. We also show that for realistic parameter values, the model generates dynamics that are broadly consistent with US data. In particular, the model generates more rapid growth of employment in less capital-intensive sectors, more rapid growth of real output in more capital-intensive sectors and aggregate behavior in line with the Kaldor facts. Finally, we construct and analyze a model of "non-balanced endogenous growth," which extends the main results of the paper to an economy with endogenous anddirected technical change. This model shows that equilibrium will typically involve endogenous non-balanced technological progress.
\end{abstract}

Daron Acemoglu

Department of Economics

MIT, E52-380B

50 Memorial Drive

Cambridge, MA 02142-1347

and NBER

daron@mit.edu

Veronica Guerrieri

University of Chicago

Graduate School of Business

5807 South Woodlawn Avenue

Room 310

Chicago, IL 60637

vguerrie@chicagogsb.edu 


\section{Introduction}

Most models of economic growth strive to be consistent with the "Kaldor facts", i.e., the relative constancy of the growth rate, the capital-output ratio, the share of capital income in GDP and the real interest rate (see Kaldor, 1963, and also Denison, 1974, Homer and Sylla, 1991, Barro and Sala-i-Martin, 2004). Beneath this balanced picture, however, are the patterns that Kongsamut, Rebelo and Xie (2001) refer to as the "Kuznets facts", which concern the systematic change in the relative importance of various sectors, in particular, agriculture, manufacturing and services (see Kuznets, 1957, 1973, Chenery, 1960, Kongsamut, Rebelo and Xie, 2001). While the Kaldor facts emphasize the balanced nature of economic growth, the Kuznets facts highlight its non-balanced nature. Figure 1 illustrates some aspects of both the Kaldor and Kuznets facts for postwar US; the capital share of national income is relatively constant, whereas relative employment and output in services increase significantly. ${ }^{1}$

The Kuznets facts have motivated a small literature, which typically starts by positing non-homothetic preferences consistent with Engel's law. ${ }^{2}$ This literature therefore emphasizes the demand-side reasons for non-balanced growth; the marginal rate of substitution between different goods changes as an economy grows, directly leading to a pattern of uneven growth between sectors. An alternative thesis, first proposed by Baumol (1967), emphasizes the potential non-balanced nature of economic growth resulting from differential productivity growth across sectors, but has received less attention in the literature. ${ }^{3}$

This paper has two aims. First, it shows that there is a natural supply-side reason, related to Baumol's (1967) thesis, for economic growth to be non-balanced. Differences in factor proportions across sectors (i.e., different shares of capital) combined with capital deepening

\footnotetext{
${ }^{1}$ All data are from the National Income and Product Accounts (NIPA). For details and definitions of services, manufacturing, employment, real GDP and capital share, see Appendix B.

${ }^{2}$ See, for example, Murphy, Shleifer and Vishny (1989), Matsuyama (1992), Echevarria (1997), Laitner (2000), Kongsamut, Rebelo and Xie (2001), Caselli and Coleman (2001), Gollin, Parente and Rogerson (2002). See also the interesting papers by Stokey (1988), Matsuyama (2002), Foellmi and Zweimuller (2002), and Buera and Kaboski (2006), which derive non-homothetiticites from the presence of a "hierarchy of needs" or "hierarchy of qualities". Finally, Hall and Jones (2006) point out that there are natural reasons for health care to be a superior good (because expected life expectancy multiplies utility) and show how this can account for the increase in health care spending. Matsuyama (2005) presents an excellent overview of this literature.

${ }^{3}$ Two exceptions are the two recent independent papers by Ngai and Pissarides (2006) and Zuleta and Young (2006). Ngai and Pissarides (2006), for example, construct a model of multi-sector economic growth inspired by Baumol. In Ngai and Pissarides's model, there are exogenous Total Factor Productivity differences across sectors, but all sectors have identical Cobb-Douglas production functions. While both of these papers are potentially consistent with the Kuznets and Kaldor facts, they do not contain the main contribution of our paper: non-balanced growth resulting from factor proportion differences and capital deepening.
} 


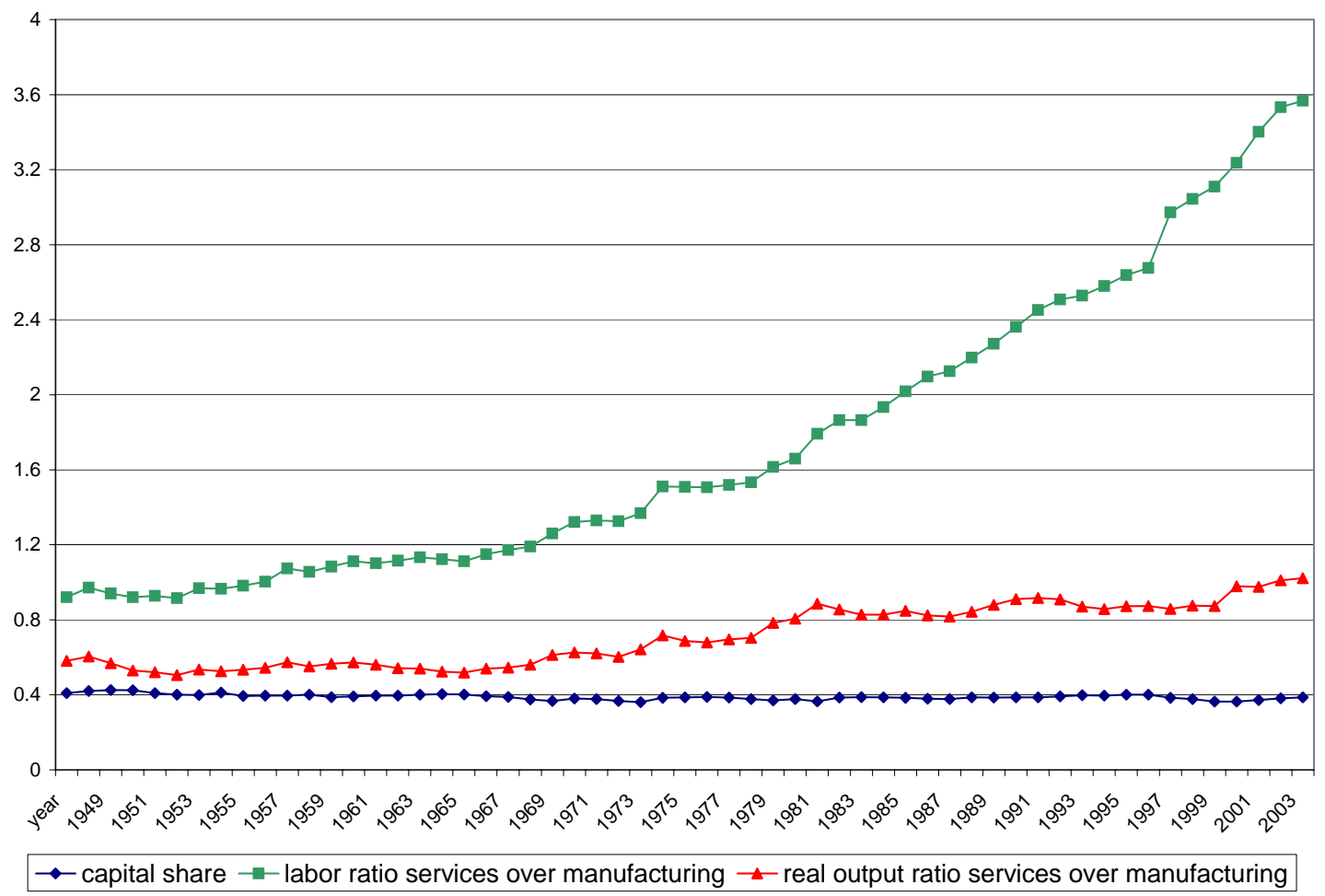

Figure 1: Capital share in national income and employment and real GDP in services relative to manufacturing in the United States, 1947-2004. Source: NIPA. See text for details.

will lead to non-balanced growth. The reason is simple: an increase in capital-labor ratio will raise output more in the sector with greater capital intensity. More specifically, we prove that "balanced technological progress", 4 capital deepening and differences in factor proportions always cause non-balanced growth. This result holds irrespective of the exact source of economic growth or the process of accumulation.

The second objective of the paper is to present and analyze a tractable two-sector growth model featuring non-balanced growth and investigate under what circumstances non-balanced growth can be consistent with aggregate Kaldor facts. We do this by constructing a class of economies with constant elasticity of substitution between two sectors and Cobb-Douglas production functions within each sector. We characterize the equilibria in this class of economies with both exogenous and endogenous technological change. We show that the limiting (asymp-

\footnotetext{
4 "Balanced technological progress" here refers to equal rates of Hicks-neutral technical change in the two sectors. Hicks-neutral technological progress is both a natural benchmark and also the type of technological progress that the more microfounded models considered in Sections 3 and 5 will generate.
} 
totic) equilibrium takes a simple form and features constant but different growth rates in each sector, constant interest rate and constant share of capital in national income.

Other properties of the limiting equilibrium of this class of economies depend on whether the products of the two sectors are gross substitutes or complements (meaning whether the elasticity of substitution between these products is greater than or less than one). Suppose for this discussion that the rates of technological progress in the two sectors are similar. In this case, when the two sectors are gross substitutes, the more capital-intensive sector dominates the economy. The form of the equilibrium is more subtle and interesting when the elasticity of substitution between these products is less than one; the growth rate of the economy is now determined by the more slowly growing, less capital-intensive sector. Despite the change in the terms of trade against the faster growing, capital-intensive sector, in equilibrium sufficient amounts of capital and labor are deployed in this sector to ensure a faster rate of growth than in the less capital-intensive sector.

One interesting feature is that our model economy generates non-balanced growth without significantly deviating from the Kaldor facts. In particular, even in the limiting equilibrium both sectors grow at positive (and unequal) rates. More importantly, when the elasticity of substitution is less than one ${ }^{5}$ convergence to this limiting equilibrium is typically slow and along the transition path, growth is non-balanced, while capital share and interest rate vary by relatively small amounts. Therefore, the equilibrium with an elasticity of substitution less than one may be able to rationalize both non-balanced sectoral growth and the Kaldor facts.

Finally, we present and analyze a model of "non-balanced endogenous growth," which shows the robustness of our results to endogenous technological progress. Our analysis shows that when sectors differ in terms of their capital intensity, equilibrium technological change will

\footnotetext{
${ }^{5}$ As we will see below, the elasticity of substitution between products will be less than one if and only if the (short-run) elasticity of substitution between labor and capital is less than one. In view of the time-series and cross-industry evidence, a short-run elasticity of substitution between labor and capital less than one appears reasonable.

For example, Hamermesh (1993), Nadiri (1970) and Nerlove (1967) survey a range of early estimates of the elasticity of substitution, which are generally between 0.3 and 0.7. David and Van de Klundert (1965) similarly estimate this elasticity to be in the neighborhood of 0.3. Using the translog production function, Griffin and Gregory (1976) estimate elasticities of substitution for nine OECD economies between 0.06 and 0.52. Berndt (1976), on the other hand, estimates an elasticity of substitution equal to 1, but does not control for a time trend, creating a strong bias towards 1. Using more recent data, and various different specifications, Krusell, Ohanian, Rios-Rull, and Violante (2000) and Antras (2001) also find estimates of the elasticity significantly less than 1. Estimates implied by the response of investment to the user cost of capital also typically imply an elasticity of substitution between capital and labor significantly less than 1 (see, e.g., Chirinko, 1993, Chirinko, Fazzari and Mayer, 1999, or Mairesse, Hall and Mulkay, 1999).
} 
itself be non-balanced and will not restore balanced growth between sectors. To the best of our knowledge, despite the large literature on endogenous growth, there are no previous studies that combine endogenous technological progress and non-balanced growth. ${ }^{6}$

A variety of evidence suggests that non-homotheticities in consumption emphasized by the previous literature are indeed present and create a tendency towards non-balanced growth. Our purpose in this paper is not to argue that these demand-side factors are unimportant, but to propose and isolate an alternative supply-side force contributing to non-balanced growth and show that it is potentially quite powerful as well. Naturally, whether or not our mechanism is important in practice is an empirical question. In Section 4, we undertake a simple calibration of our benchmark model to provide a preliminary investigation of this question. As a preparation for this calibration, Figure 2 shows the equivalent of Figure 1, but with sectors divided according to their capital intensity (see Appendix B for details). This figure shows a number of important patterns: first, consistent with our qualitative predictions, there is more rapid growth of employment in less capital-intensive sectors. ${ }^{7}$ Second, also in line with our approach, the figure shows that the rate of growth of real GDP is faster in more capital-intensive sectors. Notably, this contrasts with Figure 1, which showed faster growth in both employment and real GDP for services. The opposite movements of employment and real GDP for sectors with high and low capital intensity is a distinctive feature of our approach (for the theoretically and empirically relevant case of the elasticity of substitution less than one). The simple calibration exercise in Section 4 also shows that our model economy generates equilibrium dynamics consistent with both non-balanced growth at the sectoral level and the Kaldor facts at the aggregate level. Moreover, not only the qualitative but also the quantitative implications of our model appear to be broadly consistent with the data shown in Figure 2.

The rest of the paper is organized as follows. Section 2 shows how the combination of factor proportions differences and capital deepening lead to non-balanced growth. Section 3 constructs a more specific model with a constant elasticity of substitution between two sectors,

\footnotetext{
${ }^{6}$ See, among others, Romer (1986, 1990), Lucas (1988), Rebelo (1991), Segerstrom, Anant and Dinopoulos (1990), Stokey (1991), Grossman and Helpman (1991a,b), Aghion and Howitt (1992), Jones (1995), Young (1993). Aghion and Howitt (1998) and Barro and Sala-i-Martin (2004) provide excellent introductions to endogenous growth theory. See also Acemoglu (2002) on models of directed technical change that feature endogenous, but balanced technological progress in different sectors. Acemoglu (2003) presents a model with non-balanced technological progress between two sectors, but in the limiting equilibrium both sectors grow at the same rate.

${ }^{7}$ Note also that the magnitude of changes in Figure 2 is less than those in Figure 1, which suggests that changes in the composition of demand between manufacturing and services are likely responsible for some of the changes in the sectoral composition of output experienced over the past 60 years.
} 


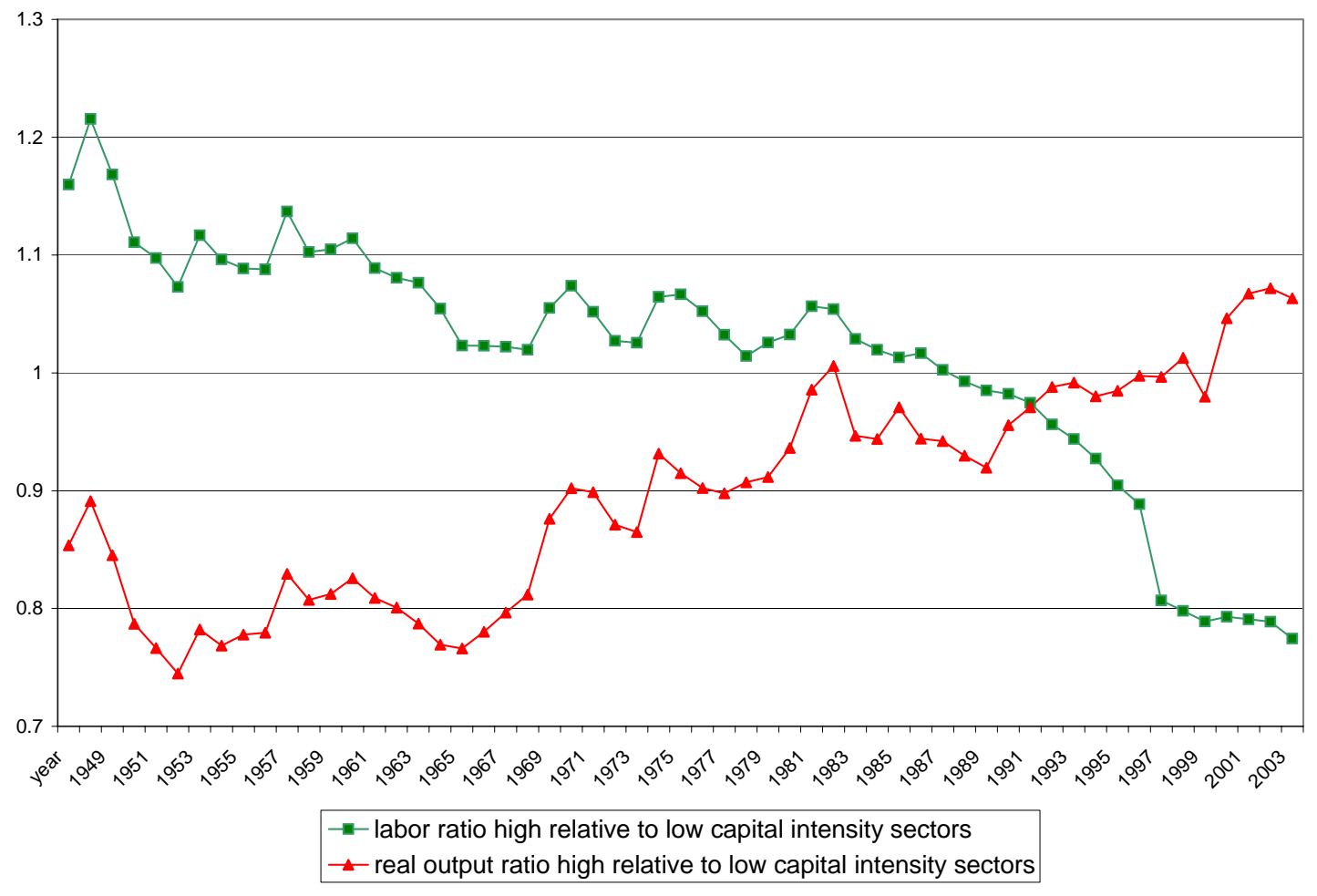

Figure 2: Capital share in national income and employment and real GDP in high capital intensity sectors relative to low capital intensity sectors, 1947-2004. Source: NIPA.

Cobb-Douglas production functions and exogenous technological progress. It characterizes the full dynamic equilibrium of this economy and shows how the model generates non-balanced sectoral growth, while remaining consistent with the Kaldor facts. Section 4 undertakes a simple calibration of our benchmark economy to investigate whether the dynamics generated by the model are consistent with the changes in the relative output and employment of capitalintensive sectors and the Kaldor facts. Section 5 introduces endogenous technological change and shows that our general results continue to hold in the presence of endogenous technological progress across sectors. Section 6 concludes. Appendix A contains proofs that are not presented in the text, while Appendix B gives some details about NIPA data and sectoral classifications used in Figures 1 and 2 and in Section 4. 


\section{Capital Deepening and Non-Balanced Growth}

We first illustrate how differences in factor proportions across sectors combined with capital deepening lead to non-balanced economic growth. To do this, we use a standard two-sector competitive model with constant returns to scale in both sectors, and two factors of production, capital, $K$, and labor, $L$. To highlight that the exact nature of the accumulation process is not essential for the results, in this section we take the sequence (process) of capital and labor supplies, $[K(t), L(t)]_{t=0}^{\infty}$, as given and assume that labor is supplied inelastically. In addition, we omit explicit time dependence when this will cause no confusion.

Final output, $Y$, is produced as an aggregate of the output of two sectors, $Y_{1}$ and $Y_{2}$,

$$
Y=F\left(Y_{1}, Y_{2}\right)
$$

and we assume that $F$ exhibits constant returns to scale and is twice continuously differentiable. Output in both sectors is produced with the production functions

$$
Y_{1}=A_{1} G_{1}\left(K_{1}, L_{1}\right)
$$

and

$$
Y_{2}=A_{2} G_{2}\left(K_{2}, L_{2}\right)
$$

The functions $G_{1}$ and $G_{2}$ also exhibit constant returns to scale and are twice continuously differentiable. $A_{1}$ and $A_{2}$ denote Hicks-neutral technology terms. Hicks-neutral technological progress is convenient to work with and is also relevant since it is the type of technological progress that the models in Sections 3 and 5 will generate. We also assume that $F$, $G_{1}$ and $G_{2}$ satisfy the Inada conditions; $\lim _{K_{j} \rightarrow 0} \partial G_{j}\left(K_{j}, L_{j}\right) / \partial K_{j}=\infty$ for all $L_{j}>0$, $\lim _{L_{j} \rightarrow 0} \partial G_{j}\left(K_{j}, L_{j}\right) / \partial L_{j}=\infty$ for all $K_{j}>0, \lim _{K_{j} \rightarrow \infty} \partial G_{j}\left(K_{j}, L_{j}\right) / \partial K_{j}=0$ for all $L_{j}<\infty, \lim _{L_{j} \rightarrow \infty} \partial G_{j}\left(K_{j}, L_{j}\right) / \partial L_{j}=0$ for all $K_{j}<\infty$, and $\lim _{Y_{j} \rightarrow 0} \partial F\left(Y_{1}, Y_{2}\right) / \partial Y_{j}=\infty$ for all $Y_{\sim j}>0$, where $j=1,2$ and $\sim j$ stands for "not $j$ ". These assumptions ensure interior solutions and simplify the exposition, though they are not necessary for the results presented in this section.

Market clearing implies

$$
\begin{aligned}
K_{1}+K_{2} & =K, \\
L_{1}+L_{2} & =L,
\end{aligned}
$$


where $K$ and $L$ are the (potentially time-varying) supplies of capital and labor, given by the exogenous sequences $[K(t), L(t)]_{t=0}^{\infty}$. We take these sequences to be continuosly differentiable functions of time. Without loss of any generality, we also ignore capital depreciation.

We normalize the price of the final good to 1 in every period and denote the prices of $Y_{1}$ and $Y_{2}$ by $p_{1}$ and $p_{2}$, and wage and rental rate of capital (interest rate) by $w$ and $r$. We assume that product and factor markets are competitive, so product prices satisfy

$$
\frac{p_{1}}{p_{2}}=\frac{\partial F\left(Y_{1}, Y_{2}\right) / \partial Y_{1}}{\partial F\left(Y_{1}, Y_{2}\right) / \partial Y_{2}}
$$

Moreover, given the Inada conditions, the wage and the interest rate satisfy: ${ }^{8}$

$$
\begin{aligned}
w & =\frac{\partial A_{1} G_{1}\left(K_{1}, L_{1}\right)}{\partial L_{1}}=\frac{\partial A_{2} G_{2}\left(K_{2}, L_{2}\right)}{\partial L_{2}} \\
r & =\frac{\partial A_{1} G_{1}\left(K_{1}, L_{1}\right)}{\partial K_{1}}=\frac{\partial A_{2} G_{2}\left(K_{2}, L_{2}\right)}{\partial K_{2}} .
\end{aligned}
$$

Definition 1 An equilibrium, given factor supply sequences, $[K(t), L(t)]_{t=0}^{\infty}$, is a sequence of product and factor prices, $\left[p_{1}(t), p_{2}(t), w(t), r(t)\right]_{t=0}^{\infty}$ and factor allocations, $\left[K_{1}(t), K_{2}(t), L_{1}(t), L_{2}(t)\right]_{t=0}^{\infty}$, such that (3), (4) and (5) are satisfied.

Let the share of capital in the two sectors be

$$
\sigma_{1} \equiv \frac{r K_{1}}{p_{1} Y_{1}} \text { and } \sigma_{2} \equiv \frac{r K_{2}}{p_{2} Y_{2}}
$$

\section{Definition 2 .}

$i$ There is capital deepening at time $t$ if $\dot{K}(t) / K(t)>\dot{L}(t) / L(t)$.

ii There are factor proportion differences at time $t$ if $\sigma_{1}(t) \neq \sigma_{2}(t)$.

iii Technological progress is balanced at time $t$ if $\dot{A}_{1}(t) / A_{1}(t)=\dot{A}_{2}(t) / A_{2}(t)$.

In this definition, $\sigma_{1}(t) \neq \sigma_{2}(t)$ refers to the equilibrium factor proportions in the two sectors at time $t$. It does not necessarily mean that these will not be equal at some future date.

The next theorem shows that if there is capital deepening and factor proportion differences, then balanced technological progress is not consistent with balanced growth.

\footnotetext{
${ }^{8}$ Without the Inada conditions, these would have to be written as $w \geq \partial A_{1} G_{1}\left(K_{1}, L_{1}\right) / \partial L_{1}$ and $L_{1} \geq 0$
}

with complementary slackness, etc. 
Theorem 1 Suppose that at time $t$, there are factor proportion differences between the two sectors, technological progress is balanced, and there is capital deepening, then growth is not balanced, that is, $\dot{Y}_{1}(t) / Y_{1}(t) \neq \dot{Y}_{2}(t) / Y_{2}(t)$.

Proof. First define the capital to labor ratio in the two sectors as

$$
k_{1} \equiv \frac{K_{1}}{L_{1}} \text { and } k_{2} \equiv \frac{K_{2}}{L_{2}}
$$

and the "per capita production functions" (without the Hicks-neutral technology term) as

$$
g_{1}\left(k_{1}\right) \equiv \frac{G_{1}\left(K_{1}, L_{1}\right)}{L_{1}} \text { and } g_{2}\left(k_{2}\right) \equiv \frac{G_{2}\left(K_{2}, L_{2}\right)}{L_{2}} .
$$

Since $G_{1}$ and $G_{2}$ are twice continuously differentiable, so are $g_{1}$ and $g_{2}$.

Now, differentiating the production functions for the two sectors,

$$
\frac{\dot{Y}_{1}}{Y_{1}}=\frac{\dot{A}_{1}}{A_{1}}+\sigma_{1} \frac{\dot{K}_{1}}{K_{1}}+\left(1-\sigma_{1}\right) \frac{L_{1}}{L_{1}}
$$

and

$$
\frac{\dot{Y}_{2}}{Y_{2}}=\frac{\dot{A}_{2}}{A_{2}}+\sigma_{2} \frac{\dot{K}_{2}}{K_{2}}+\left(1-\sigma_{2}\right) \frac{L_{2}}{L_{2}} .
$$

Suppose, to obtain a contradiction, that $\dot{Y}_{1} / Y_{1}=\dot{Y}_{2} / Y_{2}$. Since $\dot{A}_{1} / A_{1}=\dot{A}_{2} / A_{2}$ and $\sigma_{1} \neq \sigma_{2}$, this implies $\dot{k}_{1} / k_{1} \neq \dot{k}_{2} / k_{2}$. [Otherwise, $\dot{k}_{1} / k_{1}=\dot{k}_{2} / k_{2}>0$, and capital deepening implies $\dot{Y}_{1} / Y_{1} \neq \dot{Y}_{2} / Y_{2}$; for example, if $\sigma_{1}<\sigma_{2}$, then $\left.\dot{Y}_{1} / Y_{1}<\dot{Y}_{2} / Y_{2}\right]$.

Since $F$ exhibits constant returns to scale, (4) implies

$$
\frac{\dot{p}_{1}}{p_{1}}=\frac{\dot{p}_{2}}{p_{2}}=0 .
$$

Given the definition in (7), equation (5) yields the following interest rate and wage conditions:

$$
\begin{aligned}
r & =p_{1} A_{1} g_{1}^{\prime}\left(k_{1}\right) \\
& =p_{2} A_{2} g_{2}^{\prime}\left(k_{2}\right),
\end{aligned}
$$

and

$$
\begin{aligned}
w & =p_{1} A_{1}\left(g_{1}\left(k_{1}\right)-g_{1}^{\prime}\left(k_{1}\right) k_{1}\right) \\
& =p_{2} A_{2}\left(g_{2}\left(k_{2}\right)-g_{2}^{\prime}\left(k_{2}\right) k_{2}\right) .
\end{aligned}
$$


Differentiating the interest rate condition, (9), with respect to time and using (8), we obtain:

$$
\frac{\dot{A}_{1}}{A_{1}}+\varepsilon_{g_{1}^{\prime}} \frac{\dot{k}_{1}}{k_{1}}=\frac{\dot{A}_{2}}{A_{2}}+\varepsilon_{g_{2}^{\prime}} \frac{\dot{k}_{2}}{k_{2}}
$$

where

$$
\varepsilon_{g_{1}^{\prime}} \equiv \frac{g_{1}^{\prime \prime}\left(k_{1}\right) k_{1}}{g_{1}^{\prime}\left(k_{1}\right)} \text { and } \varepsilon_{g_{2}^{\prime}} \equiv \frac{g_{2}^{\prime \prime}\left(k_{2}\right) k_{2}}{g_{2}^{\prime}\left(k_{2}\right)}
$$

Since $\dot{A}_{1} / A_{1}=\dot{A}_{2} / A_{2}$

$$
\varepsilon_{g_{1}^{\prime}} \frac{\dot{k}_{1}}{k_{1}}=\varepsilon_{g_{2}^{\prime}} \frac{\dot{k}_{2}}{k_{2}}
$$

Differentiating the wage condition, (10), with respect to time, using (8) and some algebra gives:

$$
\frac{\dot{A}_{1}}{A_{1}}-\frac{\sigma_{1}}{1-\sigma_{1}} \varepsilon_{g_{1}^{\prime}} \frac{\dot{k}_{1}}{k_{1}}=\frac{\dot{A}_{2}}{A_{2}}-\frac{\sigma_{2}}{1-\sigma_{2}} \varepsilon_{g_{2}^{\prime}} \frac{\dot{k}_{2}}{k_{2}}
$$

Since $\dot{A}_{1} / A_{1}=\dot{A}_{2} / A_{2}$ and $\sigma_{1} \neq \sigma_{2}$, this equation is inconsistent with (11), yielding a contradiction and proving the claim.

The intuition for this result can be obtained as follows. Suppose that there is capital deepening and that, for concreteness, sector 2 is more capital-intensive (i.e., $\sigma_{1}<\sigma_{2}$ ). Now, if both capital and labor are allocated to the two sectors with constant proportions, the more capital-intensive sector, sector 2 , will grow faster than sector 1 . In equilibrium, the faster growth in sector 2 will naturally change equilibrium prices, and the decline in the relative price of sector 2 will cause some of the labor and capital to be reallocated to sector 1 . However, this reallocation cannot entirely offset the greater increase in the output of sector 2 , since, if it did, the relative price change that stimulated the reallocation would not take place. Consequently, equilibrium growth must be non-balanced.

The proof of Theorem 1 also makes it clear that balanced technological progress is not necessary for the result, but simply sufficient. This point is further discussed following Theorem 3 in the next section.

It is useful to relate Theorem 1 to Rybczynski's Theorem in international trade (Rybczynski, 1950). Rybczynski's Theorem states that for an open economy within the "cone of diversification" (where factor prices do not depend on factor endowments), changes in factor endowments will be absorbed by changes in sectoral output mix. Our result can be viewed as a closed-economy analogue of Rybczynski's Theorem; it shows that changes in factor endowments (capital deepening) will be absorbed by faster growth in one sector than the other, 
even though relative prices of goods and factors will change in response to the change in factor endowments. ${ }^{9}$

Finally, the proof of Theorem 1 makes it clear that the two-sector structure is not necessary for this result. In light of this, we also state a generalization for $N \geq 2$ sectors, where aggregate output is given by the constant returns to scale production function

$$
Y=F\left(Y_{1}, Y_{2}, \ldots, Y_{N}\right)
$$

Defining $\sigma_{j}$ as the capital share in sector $j=1, \ldots, N$ as in (6), we have:

Theorem 2 Suppose that at time $t$, there are factor proportion differences among the $N$ sectors in the sense that there exists $i$ and $j \leq N$ such that $\sigma_{i}(t) \neq \sigma_{j}(t)$, technological progress is balanced between $i$ and $j$, i.e., $\dot{A}_{i}(t) / A_{i}(t)=\dot{A}_{j}(t) / A_{j}(t)$, and there is capital deepening, i.e., $\dot{K}(t) / K(t)>\dot{L}(t) / L(t)$, then growth is not balanced and $\dot{Y}_{i}(t) / Y_{i}(t) \neq \dot{Y}_{j}(t) / Y_{j}(t)$.

The proof of this theorem parallels that of Theorem 1 and is omitted.

\section{Two-Sector Growth with Exogenous Technology}

The previous section demonstrated that differences in factor proportions across sectors and capital deepening will lead to non-balanced growth. This result was proved for a given (arbitrary) sequence of capital and labor supplies, $[K(t), L(t)]_{t=0}^{\infty}$, but this level of generality does not allow us to fully characterize the equilibrium path and its limiting properties. We now wish to analyze the equilibrium behavior of such an economy fully, which requires us to endogenize the sequence of capital stocks (so that capital deepening emerges as an equilibrium phenomenon). We will accomplish this by imposing more structure both on the production technology and on preferences. Capital deepening will result from exogenous technological progress, which will in turn be endogenized in Section 5.

\footnotetext{
${ }^{9}$ Note also that even if the result in Theorem 1 holds asymptotically (as $t \rightarrow \infty$ ), it does not contradict the celebrated "Turnpike theorems" in the optimal growth literature (e.g., Radner, 1961, Scheinkman, 1976, Bewley, 1982, McKenzie, 1998, Jensen, 2002). These theorems show that starting from different initial points, the economy will tend to the same asymptotic trajectory. The fact that growth is non-balanced does not preclude this possibility.
} 


\subsection{Demographics, Preferences and Technology}

The economy consists of $L(t)$ workers at time $t$, supplying their labor inelastically. There is exponential population growth,

$$
L(t)=\exp (n t) L(0)
$$

We assume that all households have constant relative risk aversion (CRRA) preferences over total household consumption (rather than per capita consumption), and all population growth takes place within existing households (thus there is no growth in the number of households). ${ }^{10}$ This implies that the economy admits a representative agent with CRRA preferences:

$$
\int_{0}^{\infty} \frac{C(t)^{1-\theta}-1}{1-\theta} e^{-\rho t} d t
$$

where $C(t)$ is aggregate consumption at time $t, \rho$ is the rate of time preferences and $\theta \geq 0$ is the inverse of the intertemporal elasticity of substitution (or the coefficient of relative risk aversion). We again drop time arguments to simplify the notation whenever this causes no confusion. We also continue to assume that there is no depreciation of capital.

The flow budget constraint for the representative consumer is:

$$
\dot{K}=r K+w L+\Pi-C .
$$

Here $w$ is the equilibrium wage rate and $r$ is the equilibrium interest rate, $K$ and $L$ denote the total capital stock and the total labor force in the economy, and $\Pi$ is total net corporate profits received by the consumers (which will be equal to zero in this section).

The unique final good is produced by combining the output of two sectors with an elasticity of substitution $\varepsilon \in[0, \infty)$ :

$$
Y=\left[\gamma Y_{1}^{\frac{\varepsilon-1}{\varepsilon}}+(1-\gamma) Y_{2}^{\frac{\varepsilon-1}{\varepsilon}}\right]^{\frac{\varepsilon}{\varepsilon-1}},
$$

where $\gamma$ is a distribution parameter which determines the relative importance of the two goods in the aggregate production.

The resource constraint of the economy, in turn, requires consumption and investment to be less than total output, $Y=r K+w L+\Pi$, thus

$$
\dot{K}+C \leq Y
$$

\footnotetext{
${ }^{10}$ The alternative would be to specify population growth taking place at the extensive margin, in which case the discount rate of the representative agent would be $\rho-n$ rather than $\rho$, without any important changes in the analysis.
} 
The two goods $Y_{1}$ and $Y_{2}$ are produced competitively using constant elasticity of substitution (CES) production functions with elasticity of substitution between intermediates equal to $\nu>1$ :

$$
Y_{1}=\left(\int_{0}^{M_{1}} y_{1}(i)^{\frac{\nu-1}{\nu}} d i\right)^{\frac{\nu}{\nu-1}} \text { and } Y_{2}=\left(\int_{0}^{M_{2}} y_{2}(i)^{\frac{\nu-1}{\nu}} d i\right)^{\frac{\nu}{\nu-1}}
$$

where $y_{1}(i)$ 's and $y_{2}(i)$ 's denote the intermediates in the sectors that have different capital/labor ratios, and $M_{1}$ and $M_{2}$ represent the technology terms. In particular $M_{1}$ denotes the number of intermediates in sector 1 and $M_{2}$ the number of intermediate goods in sector 2. This structure is particularly useful since it can also be used for the analysis of endogenous growth in Section 5.

Intermediate goods are produced with the following Cobb-Douglas technologies

$$
y_{1}(i)=l_{1}(i)^{\alpha_{1}} k_{1}(i)^{1-\alpha_{1}} \text { and } y_{2}(i)=l_{2}(i)^{\alpha_{2}} k_{2}(i)^{1-\alpha_{2}}
$$

where $l_{1}(i)$ and $k_{1}(i)$ are labor and capital used in the production of good $i$ of sector 1 and $l_{2}(i)$ and $k_{2}(i)$ are labor and capital used in the production of good $i$ of sector $2 .^{11}$

The parameters $\alpha_{1}$ and $\alpha_{2}$ determine which sector is more "capital intensive". ${ }^{12}$ When $\alpha_{1}>\alpha_{2}$, sector 1 is less capital intensive, while the converse applies when $\alpha_{1}<\alpha_{2}$. In the rest of the analysis, we assume that

$$
\alpha_{1}>\alpha_{2}
$$

which only rules out the case where $\alpha_{1}=\alpha_{2}$, since the two sectors are otherwise identical and the labeling of the sector with the lower capital share as sector 1 is without loss of any generality.

All factor markets are competitive, and market clearing for the two factors imply

$$
\int_{0}^{M_{1}} l_{1}(i) d i+\int_{0}^{M_{2}} l_{2}(i) d i \equiv L_{1}+L_{2}=L
$$

and

$$
\int_{0}^{M_{1}} k_{1}(i) d i+\int_{0}^{M_{2}} k_{2}(i) d i \equiv K_{1}+K_{2}=K
$$

\footnotetext{
${ }^{11}$ Strictly speaking, we should have two indices, $i_{1} \in \mathcal{M}_{1}$ and $i_{2} \in \mathcal{M}_{2}$, where $\mathcal{M}_{j}$ is the set of intermediates of type $j$, and $M_{j}$ is the measure of the set $\mathcal{M}_{j}$. We simplify the notation by using a generic $i$ to denote both indices, and let the context determine which index is being referred to.

${ }^{12}$ We use the term "capital intensive" as corresponding to a greater share of capital in value added, i.e., $\sigma_{2}>\sigma_{1}$ in terms of the notation of the previous section. While this share is constant because of the CobbDouglas technologies, the equilibrium ratios of capital to labor in the two sectors depend on prices.
} 
where the first set of equalities in these equations define $K_{1}, L_{1}, K_{2}$ and $L_{2}$ as the levels of capital and labor used in the two sectors, and the second set of equalities impose market clearing.

The number of intermediate goods in the two sectors evolve at the exogenous rates

$$
\frac{\dot{M}_{1}}{M_{1}}=m_{1} \text { and } \frac{\dot{M}_{2}}{M_{2}}=m_{2} .
$$

Since $M_{1}$ and $M_{2}$ determine productivity in their respective sectors, we will refer to them as "technology". In this section, we assume that all intermediates are also produced competitively (i.e., any firm can produce any of the existing intermediates). In Section 5, we will modify this assumption and assume that new intermediates are invented by R\&D and the firm that invents a new intermediate has a fully-enforced perpetual patent for its production.

\subsection{Equilibrium}

Recall that $w$ and $r$ denote the wage and the capital rental rate, and $p_{1}$ and $p_{2}$ denote the prices of the $Y_{1}$ and $Y_{2}$ goods, with the price of the final good normalized to one. Let $\left[q_{1}(i)\right]_{i=1}^{M_{1}}$ and $\left[q_{2}(i)\right]_{i=1}^{M_{2}}$ be the prices for labor-intensive and capital-intensive intermediates.

Definition 3 An equilibrium is given by paths for factor, intermediate and final goods prices $r, w,\left[q_{1}(i)\right]_{i=1}^{M_{1}},\left[q_{2}(i)\right]_{i=1}^{M_{2}}, p_{1}$ and $p_{2}$, employment and capital allocation $\left[l_{1}(i)\right]_{i=1}^{M_{1}},\left[l_{2}(i)\right]_{i=1}^{M_{2}}$, $\left[k_{1}(i)\right]_{i=1}^{M_{1}},\left[k_{2}(i)\right]_{i=1}^{M_{2}}$ such that firms maximize profits and markets clear, and consumption and savings decisions, $C$ and $\dot{K}$, maximize consumer utility.

It is useful to break the characterization of equilibrium into two pieces: static and dynamic. The static part takes the state variables of the economy, which are the capital stock, the labor supply and the technology, $K, L, M_{1}$ and $M_{2}$, as given and determines the allocation of capital and labor across sectors and factor and good prices. The dynamic part of the equilibrium determines the evolution of the endogenous state variable, $K$ (the dynamic behavior of $L$ is given by (12) and those of $M_{1}$ and $M_{2}$ by (21)).

Our choice of numeraire implies that the price of the final good, $P$, satisfies:

$$
1 \equiv P=\left[\gamma^{\varepsilon} p_{1}^{1-\varepsilon}+(1-\gamma)^{\varepsilon} p_{2}^{1-\varepsilon}\right]^{\frac{1}{1-\varepsilon}}
$$


Next, since $Y_{1}$ and $Y_{2}$ are supplied competitively, their prices are equal to the value of their marginal product, thus

$$
p_{1}=\gamma\left(\frac{Y_{1}}{Y}\right)^{-\frac{1}{\varepsilon}} \quad \text { and } \quad p_{2}=(1-\gamma)\left(\frac{Y_{2}}{Y}\right)^{-\frac{1}{\varepsilon}}
$$

and the demands for intermediates, $y_{1}(i)$ and $y_{2}(i)$, are given by the familiar isoelastic demand curves:

$$
\frac{q_{1}(i)}{p_{1}}=\left(\frac{y_{1}(i)}{Y_{1}}\right)^{-\frac{1}{\nu}} \quad \text { and } \quad \frac{q_{2}(i)}{p_{2}}=\left(\frac{y_{2}(i)}{Y_{2}}\right)^{-\frac{1}{\nu}}
$$

Since all intermediates are produced competitively, prices must equal marginal cost. Moreover, given the production functions in (18), the marginal costs of producing intermediates take the familiar Cobb-Douglas form,

$$
m c_{1}(i)=\alpha_{1}^{-\alpha_{1}}\left(1-\alpha_{1}\right)^{\alpha_{1}-1} r^{1-\alpha_{1}} w^{\alpha_{1}} \text { and } m c_{2}(i)=\alpha_{2}^{-\alpha_{2}}\left(1-\alpha_{2}\right)^{\alpha_{2}-1} r^{1-\alpha_{2}} w^{\alpha_{2}}
$$

Therefore, at all points in time, intermediate prices satisfy

$$
q_{1}(i)=\alpha_{1}\left(1-\alpha_{1}\right)^{\alpha_{1}-1} r^{1-\alpha_{1}} w^{\alpha_{1}}
$$

and

$$
q_{2}(i)=\alpha_{2}\left(1-\alpha_{2}\right)^{\alpha_{2}-1} r^{1-\alpha_{2}} w^{\alpha_{2}}
$$

for all $i$.

Equations (24) and (25) imply that all intermediates in each sector sell at the same price $q_{1}=q_{1}(i)$ for all $i \leq M_{1}$ and $q_{2}=q_{2}(i)$ for all $i \leq M_{2}$. This combined with (23) implies that the demand for, and the production of, the same type of intermediate will be the same. Thus:

$$
\begin{array}{rlrl}
y_{1}(i) & =l_{1}(i)^{\alpha_{1}} k_{1}(i)^{1-\alpha_{1}} & \\
& =l_{1}^{\alpha_{1}} k_{1}^{1-\alpha_{1}}=y_{1} \quad \forall i \leq M_{1} \\
y_{2}(i) & =l_{2}(i)^{\alpha_{2}} k_{2}(i)^{1-\alpha_{2}} & \\
& =l_{2}^{\alpha_{2}} k_{2}^{1-\alpha_{2}}=y_{2} \quad \forall i \leq M_{2},
\end{array}
$$

where $l_{1}$ and $k_{1}$ are the levels of employment and capital used in each intermediate of sector 1 , and $l_{2}$ and $k_{2}$ are the levels of employment and capital used for intermediates in sector 2.

Market clearing conditions, (19) and (20), then imply that $l_{1}=L_{1} / M_{1}, k_{1}=K_{1} / M_{1}$, $l_{2}=L_{2} / M_{2}$ and $k_{2}=K_{2} / M_{2}$, so we have the output of each intermediate in the two sectors as

$$
y_{1}=\frac{L_{1}^{\alpha_{1}} K_{1}^{1-\alpha_{1}}}{M_{1}} \quad \text { and } \quad y_{2}=\frac{L_{2}^{\alpha_{2}} K_{2}^{1-\alpha_{2}}}{M_{2}} .
$$


Substituting (26) into (17), we obtain the total supply of labor- and capital-intensive goods as

$$
Y_{1}=M_{1}^{\frac{1}{\nu-1}} L_{1}^{\alpha_{1}} K_{1}^{1-\alpha_{1}} \text { and } Y_{2}=M_{2}^{\frac{1}{\nu-1}} L_{2}^{\alpha_{2}} K_{2}^{1-\alpha_{2}}
$$

Comparing these (derived) production functions to (1) and (2) highlights that in this economy, the production functions $G_{1}$ and $G_{2}$ from the previous section take Cobb-Douglas forms, with one sector always having a higher share of capital than the other sector, and also that $A_{1} \equiv M_{1}^{\frac{1}{\nu-1}}$ and $A_{2} \equiv M_{2}^{\frac{1}{\nu-1}}$.

In addition, combining (27) with (15) implies that the aggregate output of the economy is:

$$
Y=\left[\gamma\left(M_{1}^{\frac{1}{\nu-1}} L_{1}^{\alpha_{1}} K_{1}^{1-\alpha_{1}}\right)^{\frac{\varepsilon-1}{\varepsilon}}+(1-\gamma)\left(M_{2}^{\frac{1}{\nu-1}} L_{2}^{\alpha_{2}} K_{2}^{1-\alpha_{2}}\right)^{\frac{\varepsilon-1}{\varepsilon}}\right]^{\frac{\varepsilon}{\varepsilon-1}}
$$

Finally, factor prices and the allocation of labor and capital between the two sectors are determined by: ${ }^{13}$

$$
\begin{gathered}
w=\gamma \alpha_{1}\left(\frac{Y}{Y_{1}}\right)^{\frac{1}{\varepsilon}} \frac{Y_{1}}{L_{1}} \\
w=(1-\gamma) \alpha_{2}\left(\frac{Y}{Y_{2}}\right)^{\frac{1}{\varepsilon}} \frac{Y_{2}}{L_{2}} \\
r=\gamma\left(1-\alpha_{1}\right)\left(\frac{Y}{Y_{1}}\right)^{\frac{1}{\varepsilon}} \frac{Y_{1}}{K_{1}} \\
r=(1-\gamma)\left(1-\alpha_{2}\right)\left(\frac{Y}{Y_{2}}\right)^{\frac{1}{\varepsilon}} \frac{Y_{2}}{K_{2}} .
\end{gathered}
$$

These factor prices take the familiar form, equal to the marginal product of a factor from (28).

\subsection{Static Equilibrium: Comparative Statics}

Let us now analyze how changes in the state variables, $L, K, M_{1}$ and $M_{2}$, impact on equilibrium factor prices and factor shares. As noted in the Introduction, the case with $\varepsilon<1$ is of greater interest (and empirically more relevant as pointed out in footnote 5), so, throughout, we focus on this case (though we give the result for the case in which $\varepsilon>1$, and we only omit the standard case with $\varepsilon=1$ to avoid repetition).

\footnotetext{
${ }^{13}$ To obtain these equations, start with the cost functions above, and derive the demand for factors by using Shepherd's Lemma. For example, for the sector 1 , these are $l_{1}=\left(\frac{\alpha_{1}}{1-\alpha_{1}} \frac{r}{w}\right)^{1-\alpha_{1}} y_{1} \quad$ and $\quad k_{1}=\left(\frac{\alpha_{1}}{1-\alpha_{1}} \frac{r}{w}\right)^{-\alpha_{1}} y_{1}$. Combine these two equations to derive the equilibrium relationship between $r$ and $w$. Then using equation (24), eliminate $r$ to obtain a relationship between $w$ and $q_{1}$. Finally, combining this relationship with the demand curve in (23), the market clearing conditions, (19) and (20), and using (27) yields (29). The other equations are obtained similarly.
} 
Let us denote the fraction of capital and labor employed in the labor-intensive sector by $\kappa \equiv K_{1} / K$ and $\lambda \equiv L_{1} / L$ (clearly $1-\kappa \equiv K_{2} / K$ and $1-\lambda \equiv L_{2} / L$ ). Combining equations (29), (30), (31) and (32), we obtain:

$$
\kappa=\left[1+\left(\frac{1-\alpha_{2}}{1-\alpha_{1}}\right)\left(\frac{1-\gamma}{\gamma}\right)\left(\frac{Y_{1}}{Y_{2}}\right)^{\frac{1-\varepsilon}{\varepsilon}}\right]^{-1}
$$

and

$$
\lambda=\left[\left(\frac{1-\alpha_{1}}{1-\alpha_{2}}\right)\left(\frac{\alpha_{2}}{\alpha_{1}}\right)\left(\frac{1-\kappa}{\kappa}\right)+1\right]^{-1} .
$$

Equation (34) makes it clear that the share of labor in sector $1, \lambda$, is monotonically increasing in the share of capital in sector $1, \kappa$. We next determine how these two shares change with capital accumulation and technological change.

Proposition 1 In equilibrium,

1.

$$
\frac{d \ln \kappa}{d \ln K}=-\frac{d \ln \kappa}{d \ln L}=\frac{(1-\varepsilon)\left(\alpha_{1}-\alpha_{2}\right)(1-\kappa)}{1+(1-\varepsilon)\left(\alpha_{1}-\alpha_{2}\right)(\kappa-\lambda)}>0 \Leftrightarrow\left(\alpha_{1}-\alpha_{2}\right)(1-\varepsilon)>0
$$

2.

$$
\frac{d \ln \kappa}{d \ln M_{2}}=-\frac{d \ln \kappa}{d \ln M_{1}}=\frac{(1-\varepsilon)(1-\kappa) /(\nu-1)}{1+(1-\varepsilon)\left(\alpha_{1}-\alpha_{2}\right)(\kappa-\lambda)}>0 \Leftrightarrow \varepsilon<1 .
$$

The proof of this proposition is straightforward and is omitted.

Equation (35), part 1 of the proposition, states that when the elasticity of substitution between sectors, $\varepsilon$, is less than 1 , the fraction of capital allocated to the capital-intensive sector declines in the stock of capital (and conversely, when $\varepsilon>1$, this fraction is increasing in the stock of capital). To obtain the intuition for this comparative static, which is useful for understanding many of the results that will follow, note that if $K$ increases and $\kappa$ remains constant, then the capital-intensive sector, sector 2, will grow by more than sector 1 . Equilibrium prices given in $(22)$ then imply that when $\varepsilon<1$ the relative price of the capital-intensive sector will fall more than proportionately, inducing a greater fraction of capital to be allocated to the less capital-intensive sector 1 . The intuition for the converse result when $\varepsilon>1$ is straightforward.

Moreover, equation (36) implies that when the elasticity of substitution, $\varepsilon$, is less than one, an improvement in the technology of a sector causes the share of capital going to that sector to fall. The intuition is again the same: when $\varepsilon<1$, increased production in a sector causes 
a more than proportional decline in its relative price, inducing a reallocation of capital away from it towards the other sector (again the converse results and intuition apply when $\varepsilon>1$ ).

Proposition 1 gives only the comparative statics for $\kappa$. Equation (34) immediately implies that the same comparative statics apply to $\lambda$ and thus yields the following corollary:

Corollary 1 In equilibrium,

1.

$$
\frac{d \ln \lambda}{d \ln K}=-\frac{d \ln \lambda}{d \ln L}>0 \Leftrightarrow\left(\alpha_{1}-\alpha_{2}\right)(1-\varepsilon)>0
$$

2.

$$
\frac{d \ln \lambda}{d \ln M_{2}}=-\frac{d \ln \lambda}{d \ln M_{1}}>0 \Leftrightarrow \varepsilon<1
$$

Next, combining (29) and (31), we also obtain relative factor prices as

$$
\frac{w}{r}=\frac{\alpha_{1}}{1-\alpha_{1}}\left(\frac{\kappa K}{\lambda L}\right)
$$

and the capital share in the economy as: ${ }^{14}$

$$
\sigma_{K} \equiv \frac{r K}{Y}=1-\gamma \alpha_{1}\left(\frac{Y_{1}}{Y}\right)^{\frac{\varepsilon-1}{\varepsilon}} \lambda^{-1}
$$

Proposition 2 In equilibrium,

1.

$$
\frac{d \ln (w / r)}{d \ln K}=-\frac{d \ln (w / r)}{d \ln L}=\frac{1}{1+(1-\varepsilon)\left(\alpha_{1}-\alpha_{2}\right)(\kappa-\lambda)}>0
$$

2.

$$
\frac{d \ln (w / r)}{d \ln M_{2}}=-\frac{d \ln (w / r)}{d \ln M_{1}}=-\frac{(1-\varepsilon)(\kappa-\lambda) /(\nu-1)}{1+(1-\varepsilon)\left(\alpha_{1}-\alpha_{2}\right)(\kappa-\lambda)}<0 \Leftrightarrow\left(\alpha_{1}-\alpha_{2}\right)(1-\varepsilon)>0 .
$$

3.

$$
\frac{d \ln \sigma_{K}}{d \ln K}<0 \Leftrightarrow \varepsilon<1
$$

4.

$$
\frac{d \ln \sigma_{K}}{d \ln M_{2}}=-\frac{d \ln \sigma_{K}}{d \ln M_{1}}<0 \Leftrightarrow\left(\alpha_{1}-\alpha_{2}\right)(1-\varepsilon)>0 .
$$

\footnotetext{
${ }^{14}$ Note that $\sigma_{K}$ refers to the share of capital in national income, and is thus different from the capital shares in the previous section, which were sector specific. Sector-specific capital shares are constant here because of the Cobb-Douglas production functions (in particular, $\sigma_{1}=\alpha_{1}$ and $\sigma_{2}=\alpha_{2}$ ).
} 
Proof. Parts 1 and 2 follow from differentiating equation (37) and Proposition 1. Here we prove parts 3 and 4 . First, combine the production function and the equilibrium capital allocation to obtain

$$
\begin{aligned}
\left(\frac{Y_{1}}{Y}\right)^{\frac{\varepsilon-1}{\varepsilon}} & =\left[\gamma+(1-\gamma)\left(\frac{Y_{1}}{Y_{2}}\right)^{\frac{1-\varepsilon}{\varepsilon}}\right]^{-1} \\
& =\gamma^{-1}\left(1+\left(\frac{1-\alpha_{1}}{1-\alpha_{2}}\right)\left(\frac{1}{\kappa}-1\right)\right)^{-1}
\end{aligned}
$$

Then, using the results of Proposition 1 and the definition of $\sigma_{K}$ from (38), we have:

$$
\frac{d \ln \sigma_{K}}{d \ln K}=-S\left(\frac{1-\sigma_{K}}{\sigma_{K}}\right)\left(\frac{1-\alpha_{1}}{1-\alpha_{2}}\right) \frac{(1-\varepsilon)\left(\alpha_{1}-\alpha_{2}\right)(1-\kappa) / \kappa}{1+(1-\varepsilon)\left(\alpha_{1}-\alpha_{2}\right)(\kappa-x)}
$$

and

$$
\frac{d \ln \sigma_{K}}{d \ln M_{2}}=-\frac{d \ln \sigma_{K}}{d \ln M_{1}}=S\left(\frac{1-\sigma_{K}}{\sigma_{K}}\right)\left(\frac{1-\alpha_{1}}{1-\alpha_{2}}\right) \frac{(1-\varepsilon)(1-\kappa) / \kappa(\nu-1)}{1+(1-\varepsilon)\left(\alpha_{1}-\alpha_{2}\right)(\kappa-x)},
$$

where

$$
S \equiv\left[\left(1+\left(\frac{1-\alpha_{1}}{1-\alpha_{2}}\right)\left(\frac{1}{\kappa}-1\right)\right)^{-1}-\left(\left(\frac{\alpha_{1}}{\alpha_{2}}\right)+\left(\frac{1-\alpha_{1}}{1-\alpha_{2}}\right)\left(\frac{1}{\kappa}-1\right)\right)^{-1}\right]
$$

Clearly,

$$
S<0 \Leftrightarrow \alpha_{1}>\alpha_{2}
$$

Equations (41) and (42) then imply (39) and (40).

The most important result in this proposition is part 3, which links the equilibrium relationship between the capital share in national income and the capital stock to the elasticity of substitution. Since a negative relationship between the share of capital in national income and the capital stock is equivalent to capital and labor being gross complements in the aggregate, this result also implies that, as claimed in footnote 5, the elasticity of substitution between capital and labor is less than one if and only if $\varepsilon$ is less than one. Intuitively, as in Theorem 1 , an increase in the capital stock of the economy causes the output of the more capital-intensive sector, sector 2, to increase relative to the output in the less capital-intensive sector (despite the fact that the share of capital allocated to the less-capital intensive sector increases as shown in equation (35)). This then increases the production of the more capital-intensive sector, and, when $\varepsilon<1$, it reduces the relative reward to capital (and the share of capital in national income). The converse result applies when $\varepsilon>1$.

Moreover, when $\varepsilon<1$, part 4 implies that an increase in $M_{1}$ is "capital biased" and an increase in $M_{2}$ is "labor biased". The intuition for why an increase in the productivity of the 
sector that is intensive in capital is biased toward labor (and vice versa) is once again similar: when the elasticity of substitution between the two sectors, $\varepsilon$, is less than one, an increase in the output of a sector (this time driven by a change in technology) decreases its price more than proportionately, thus reducing the relative compensation of the factor used more intensively in that sector (see Acemoglu, 2002). When $\varepsilon>1$, we have the converse pattern, and an increase in $M_{2}$ is "capital biased," while an increase in $M_{1}$ is "labor biased"

\subsection{Dynamic Equilibrium}

We now turn to the characterization of the dynamic equilibrium path of this economy. We start with the Euler equation for consumers, which follows from the maximization of (13). The Euler equation takes the familiar form:

$$
\frac{\dot{C}}{C}=\frac{1}{\theta}(r-\rho)
$$

Since the only asset of the representative household in this economy is capital, the transversality condition takes the standard form:

$$
\lim _{t \rightarrow \infty} K(t) \exp \left(-\int_{0}^{t} r(\tau) d \tau\right)=0,
$$

which, together with the Euler equation (43) and the resource constraint (16), determines the dynamic behavior of consumption and capital stock, $C$ and $K$. Equations (12) and (21) give the behavior of $L, M_{1}$ and $M_{2}$.

We can therefore define a dynamic equilibrium as follows:

Definition 4 A dynamic equilibrium is given by paths of wages, interest rates, labor and capital allocation decisions, $w, r, \lambda$ and $\kappa$, satisfying (29), (30), (31), (32), (33) and (34), and of consumption, $C$, capital stock, $K$, employment, $L$, and technology, $M_{1}$ and $M_{2}$, satisfying (12), (16), (21), (43), and (44).

Let us also introduce the following notation for growth rates of the key objects in this economy:

$$
\begin{aligned}
& \frac{\dot{L}_{1}}{L_{1}} \equiv n_{1}, \frac{\dot{L}_{2}}{L_{2}} \equiv n_{2}, \frac{\dot{L}}{L} \equiv n, \\
& \frac{\dot{K}_{1}}{K_{1}} \equiv z_{1}, \frac{\dot{K}_{2}}{K_{2}} \equiv z_{2}, \frac{\dot{K}}{K} \equiv z
\end{aligned}
$$




$$
\frac{\dot{Y}_{1}}{Y_{1}} \equiv g_{1}, \frac{\dot{Y}_{2}}{Y_{2}} \equiv g_{2}, \frac{\dot{Y}}{Y} \equiv g
$$

so that $n_{s}$ and $z_{s}$ denote the growth rate of labor and capital stock, $m_{s}$ denotes the growth rate of technology, and $g_{s}$ denotes the growth rate of output in sector $s$. Moreover, whenever they exist, we denote the corresponding asymptotic growth rates by asterisks, i.e.,

$$
n_{s}^{*}=\lim _{t \rightarrow \infty} n_{s}, z_{s}^{*}=\lim _{t \rightarrow \infty} z_{s} \text { and } g_{s}^{*}=\lim _{t \rightarrow \infty} g_{s}
$$

Similarly denote the asymptotic capital and labor allocation decisions by asterisks

$$
\kappa^{*}=\lim _{t \rightarrow \infty} \kappa \text { and } \lambda^{*}=\lim _{t \rightarrow \infty} \lambda .
$$

We now state and prove two lemmas that will be useful both in this section and again in Section 5 .

Lemma 1 If $\varepsilon<1$, then $n_{1} \gtreqless n_{2} \Leftrightarrow z_{1} \gtreqless z_{2} \Leftrightarrow g_{1} \lesseqgtr g_{2}$. If $\varepsilon>1$, then $n_{1} \gtreqless n_{2} \Leftrightarrow z_{1} \gtreqless z_{2} \Leftrightarrow$ $g_{1} \gtreqless g_{2}$.

Proof. Differentiating (29) and (30) with respect to time yields

$$
\frac{\dot{w}}{w}+n_{1}=\frac{1}{\varepsilon} g+\frac{\varepsilon-1}{\varepsilon} g_{1} \text { and } \frac{\dot{w}}{w}+n_{2}=\frac{1}{\varepsilon} g+\frac{\varepsilon-1}{\varepsilon} g_{2} .
$$

Subtracting the second expression from the first gives $n_{1}-n_{2}=(\varepsilon-1)\left(g_{1}-g_{2}\right) / \varepsilon$, and immediately implies the first part of the desired result. Similarly differentiating (31) and (32) yields

$$
\frac{\dot{r}}{r}+z_{1}=\frac{1}{\varepsilon} g+\frac{\varepsilon-1}{\varepsilon} g_{1} \text { and } \frac{\dot{r}}{r}+z_{2}=\frac{1}{\varepsilon} g+\frac{\varepsilon-1}{\varepsilon} g_{2} .
$$

Subtracting the second expression from the first again gives the second part of the result.

This lemma establishes the straightforward, but at first counter-intuitive, result that, when the elasticity of substitution between the two sectors is less than one, the equilibrium growth rate of the capital stock and labor force in the sector that is growing faster must be less than in the other sector. When the elasticity of substitution is greater than one, the converse result obtains. To see the intuition, note that terms of trade (relative prices) shift in favor of the more slowly growing sector. When the elasticity of substitution is less than one, this change in relative prices is more than proportional with the change in quantities and this encourages more of the factors to be allocated towards the more slowly growing sector. 
Lemma 2 Suppose the asymptotic growth rates $g_{1}^{*}$ and $g_{2}^{*}$ exist. If $\varepsilon<1$, then $g^{*}=$ $\min \left\{g_{1}^{*}, g_{2}^{*}\right\}$. If $\varepsilon>1$, then $g^{*}=\max \left\{g_{1}^{*}, g_{2}^{*}\right\}$.

Proof. Differentiating the production function for the final good (28) we obtain:

$$
g=\frac{\left[\gamma Y_{1}^{\frac{\varepsilon-1}{\varepsilon}} g_{1}+(1-\gamma) Y_{2}^{\frac{\varepsilon-1}{\varepsilon}} g_{2}\right]}{\left[\gamma Y_{1}^{\frac{\varepsilon-1}{\varepsilon}}+(1-\gamma) Y_{2}^{\frac{\varepsilon-1}{\varepsilon}}\right]}
$$

This equation, combined with $\varepsilon<1$, implies that as $t \rightarrow \infty, g^{*}=\min \left\{g_{1}^{*}, g_{2}^{*}\right\}$. Similarly, combined with $\varepsilon>1$, it implies that as $t \rightarrow \infty, g^{*}=\max \left\{g_{1}^{*}, g_{2}^{*}\right\}$.

Consequently, when the elasticity of substitution is less than 1 , the asymptotic growth rate of aggregate output will be determined by the sector that is growing more slowly, and the converse applies when $\varepsilon>1$.

\subsection{Constant Growth Paths}

We first focus on asymptotic equilibrium paths, which are equilibrium paths that the economy tends to as $t \rightarrow \infty$. A constant growth path (CGP) is defined as an equilibrium path where the asymptotic growth rate of consumption exists and is constant, i.e.,

$$
\lim _{t \rightarrow \infty} \frac{\dot{C}}{C}=g_{C}^{*}
$$

From the Euler equation (43), this also implies that the interest rate must be asymptotically constant, i.e., $\lim _{t \rightarrow \infty} \dot{r}=0$.

To establish the existence of a CGP, we impose the following parameter restriction:

$$
\rho \geq \frac{1-\theta}{\nu-1} \max \left\{\frac{m_{1}}{\alpha_{1}}, \frac{m_{2}}{\alpha_{2}}\right\}+(1-\theta) n .
$$

This assumption ensures that the transversality condition (44) holds. ${ }^{15}$ Terms of the form $m_{1} / \alpha_{1}$ or $m_{2} / \alpha_{2}$ appear naturally in equilibrium, since they capture the "augmented" rate of technological progress. In particular, recall that associated with the technological progress, there will also be equilibrium capital deepening in each sector. The overall effect on labor productivity (and output growth) will depend on the rate of technological progress augmented

\footnotetext{
${ }^{15}$ If instead we took the discount rate of the representative household to be $\rho-n$ as noted in footnote 10 , then $n$ in (A2) would not be multiplied by $(1-\theta)$.
} 
with the rate of capital deepening. The terms $m_{1} / \alpha_{1}$ or $m_{2} / \alpha_{2}$ capture this, since a lower $\alpha_{1}$ or $\alpha_{2}$ corresponds to a greater share of capital in the relevant sector, and thus a higher rate of augmented technological progress for a given rate of Hicks-neutral technological change. In this light, Assumption A2 can be understood as implying that the augmented rate of technological progress should be low enough to satisfy the transversality condition (44).

The next theorem is the main result of this part of the paper and characterizes the relatively simple form of the CGP in the presence of non-balanced growth. Although we characterize a CGP, in the sense that aggregate output grows at a constant rate, it is noteworthy that growth is non-balanced since output, capital and employment in the two sectors grow at different rates.

Theorem 3 Suppose Assumptions A1 and A2 hold. Define $s$ and $\sim s$ such that $\frac{m_{s}}{\alpha_{s}}=$ $\min \left\{\frac{m_{1}}{\alpha_{1}}, \frac{m_{2}}{\alpha_{2}}\right\}$ and $\frac{m_{\sim s}}{\alpha_{\sim s}}=\max \left\{\frac{m_{1}}{\alpha_{1}}, \frac{m_{2}}{\alpha_{2}}\right\}$ when $\varepsilon<1$, and $\frac{m_{s}}{\alpha_{s}}=\max \left\{\frac{m_{1}}{\alpha_{1}}, \frac{m_{2}}{\alpha_{2}}\right\}$ and $\frac{m_{\sim s}}{\alpha_{\sim s}}=$ $\min \left\{\frac{m_{1}}{\alpha_{1}}, \frac{m_{2}}{\alpha_{2}}\right\}$ when $\varepsilon>1$. Then there exists a unique CGP such that

$$
\begin{gathered}
g^{*}=g_{C}^{*}=g_{s}^{*}=z_{s}^{*}=n+\frac{1}{\alpha_{s}(\nu-1)} m_{s}, \\
z_{\sim s}^{*}=n-\frac{(1-\varepsilon) m_{\sim s}}{(\nu-1)}+\frac{\left[1+\alpha_{\sim s}(1-\varepsilon)\right] m_{s}}{\alpha_{s}(\nu-1)}<g^{*}, \\
g_{\sim s}^{*}=n+\frac{\varepsilon m_{\sim s}}{(\nu-1)}+\frac{\left[1-\alpha_{\sim s}\left(1-\varepsilon \alpha_{\sim s}(1-\varepsilon)\right)\right] m_{s}}{\alpha_{s}(\nu-1)\left[1-\alpha_{\sim s}(1-\varepsilon)\right]}>g^{*}, \\
n_{s}^{*}=n \text { and } n_{\sim s}^{*}=n-\frac{(1-\varepsilon)\left(\alpha_{s} m_{\sim s}-\alpha_{\sim s} m_{s}\right)}{\alpha_{s}(\nu-1)} .
\end{gathered}
$$

Proof. We prove this proposition in three steps.

Step 1: Suppose that $\varepsilon<1$. Provided that $g_{\sim s}^{*} \geq g_{s}^{*}>0$, then there exists a unique CGP defined by equations (48), (49), (50) and (51) satisfying $g_{\sim s}^{*}>g_{s}^{*}>0$, where $\frac{m_{s}}{\alpha_{s}}=$ $\min \left\{\frac{m_{1}}{\alpha_{1}}, \frac{m_{2}}{\alpha_{2}}\right\}$ and $\frac{m_{\sim s}}{\alpha_{\sim s}}=\max \left\{\frac{m_{1}}{\alpha_{1}}, \frac{m_{2}}{\alpha_{2}}\right\}$.

Step 2: Suppose that $\varepsilon>1$. Provided that $g_{\sim s}^{*} \leq g_{s}^{*}<0$, then there exists a unique CGP defined by equations (48), (49), (50) and (51) satisfying $g_{\sim s}^{*}<g_{s}^{*}<0$, where $\frac{m_{s}}{\alpha_{s}}=$ $\max \left\{\frac{m_{1}}{\alpha_{1}}, \frac{m_{2}}{\alpha_{2}}\right\}$ and $\frac{m_{\sim s}}{\alpha_{\sim s}}=\min \left\{\frac{m_{1}}{\alpha_{1}}, \frac{m_{2}}{\alpha_{2}}\right\}$.

Step 3: Any CGP must satisfy $g_{\sim s}^{*} \geq g_{s}^{*}>0$, when $\varepsilon<1$ and $g_{s}^{*} \geq g_{\sim s}^{*}>0$, when $\varepsilon>1$ with $\frac{m_{s}}{\alpha_{s}}$ defined as in the theorem.

The third step then implies that the growth rates characterized in steps 1 and 2 are indeed equilibria and there cannot be any other CGP equilibria, completing the proof. 
Proof of Step 1. Let us assume without any loss of generality that $s=1$, i.e., $\frac{m_{s}}{\alpha_{s}}=\frac{m_{1}}{\alpha_{1}}$. Given $g_{2}^{*} \geq g_{1}^{*}>0$, equations (33) and (34) imply condition that $\lambda^{*}=\kappa^{*}=1$ [in the case where $s=2$, we would have $\left.\lambda^{*}=\kappa^{*}=0\right]$ and Lemma 2 implies that we must also have $g^{*}=g_{1}^{*}$. This condition together with our system of equations, (27), (45) and (46), solves uniquely for $n_{1}^{*}, n_{2}^{*}, z_{1}^{*}, z_{2}^{*}, g_{1}^{*}$ and $g_{2}^{*}$ as given in equations (48), (49), (50) and (51). Note that this solution is consistent with $g_{2}^{*}>g_{1}^{*}>0$, since Assumptions A1 and A2 imply that $g_{2}^{*}>g_{1}^{*}$ and $g_{1}^{*}>0$. Finally, $C \leq Y,(14)$ and (44) imply that the consumption growth rate, $g_{C}^{*}$, is equal to the growth rate of output, $g^{*}$. [Suppose not, then since $C / Y \rightarrow 0$ as $t \rightarrow \infty$, the budget constraint (14) implies that asymptotically $\dot{K}(t)=Y(t)$, and integrating the budget constraint gives $K(t) \rightarrow \int_{0}^{t} Y(s) d s$, implying that the capital stock grows more than exponentially, since $Y$ is growing exponentially; this would naturally violate the transversality condition (44)].

Finally, we can verify that an equilibrium with $z_{1}^{*}, z_{2}^{*}, m_{1}^{*}, m_{2}^{*}, g_{1}^{*}$ and $g_{2}^{*}$ satisfies the transversality condition (44). Note that the transversality condition (44) will be satisfied if

$$
\lim _{t \rightarrow \infty} \frac{\dot{K}(t)}{K(t)}<r^{*}
$$

where $r^{*}$ is the constant asymptotic interest rate. Since from the Euler equation (43) $r^{*}=$ $\theta g^{*}+\rho,(52)$ will be satisfied when $g^{*}(1-\theta)<\rho$. Assumption A2 ensures that this is the case with $g^{*}=n+\frac{1}{\alpha_{1}(\nu-1)} m_{1}$. A similar argument applies for the case where $\frac{m_{s}}{\alpha_{s}}=\frac{m_{2}}{\alpha_{2}}$.

Proof of Step 2. The proof of this step is similar to the previous one, and is thus omitted.

Proof of Step 3. We now prove that along all CGPs $g_{\sim s}^{*} \geq g_{s}^{*}>0$, when $\varepsilon<1$ and $g_{s}^{*} \geq g_{\sim s}^{*}>0$, when $\varepsilon>1$ with $\frac{m_{s}}{\alpha_{s}}$ defined as in the theorem. Without any loss of generality, suppose that $\frac{m_{s}}{\alpha_{s}}=\frac{m_{1}}{\alpha_{1}}$. We now separately derive a contradiction for two configurations, (1) $g_{1}^{*} \geq g_{2}^{*}$, or $(2) g_{2}^{*} \geq g_{1}^{*}$ but $g_{1}^{*} \leq 0$.

1. Suppose $g_{1}^{*} \geq g_{2}^{*}$ and $\varepsilon<1$. Then, following the same reasoning as in Step 1 , the unique solution to the equilibrium conditions (27), (45) and (46), when $\varepsilon<1$ is:

$$
\begin{gathered}
g^{*}=g_{C}^{*}=g_{2}^{*}=z_{2}^{*}=n+\frac{1}{\alpha_{2}(\nu-1)} m_{2}, \\
z_{1}^{*}=n-\frac{(1-\varepsilon) m_{1}}{(\nu-1)}+\frac{\left[1+\alpha_{1}(1-\varepsilon)\right] m_{2}}{\alpha_{2}(\nu-1)}, \\
g_{1}^{*}=n+\frac{\varepsilon m_{1}}{(\nu-1)}+\frac{\left[1-\alpha_{1}\left(1-\varepsilon \alpha_{1}(1-\varepsilon)\right)\right] m_{2}}{\alpha_{2}(\nu-1)\left[1-\alpha_{1}(1-\varepsilon)\right]}, \\
n_{1}^{*}=n+\frac{(1-\varepsilon)\left[\alpha_{1} m_{2}-\alpha_{2} m_{1}\right]}{\alpha_{2}(\nu-1)} .
\end{gathered}
$$


Combining these equations implies that $g_{1}^{*}<g_{2}^{*}$, which contradicts the hypothesis $g_{1}^{*} \geq$ $g_{2}^{*}>0$. The argument for $\varepsilon>1$ is analogous.

2. Suppose $g_{2}^{*} \geq g_{1}^{*}$ and $\varepsilon<1$, then the same steps as above imply that there is a unique solution to equilibrium conditions (27), (45) and (46), which are given by equations (48), (49), (50) and (51). But now (48) directly contradicts $g_{1}^{*} \leq 0$. Finally suppose $g_{2}^{*} \geq g_{1}^{*}$ and $\varepsilon>1$, then the unique solution is given by (53), (54), (55) and (56). But in this case, (55) directly contradicts the hypothesis that $g_{1}^{*} \leq 0$, completing the proof.

There are a number of important implications of this theorem. First, as long as $m_{1} / \alpha_{1} \neq$ $m_{2} / \alpha_{2}$, growth is non-balanced. The intuition for this result is the same as Theorem 1 in the previous section. Suppose, for concreteness, that $m_{1} / \alpha_{1}<m_{2} / \alpha_{2}$ (which would be the case, for example, if $m_{1} \approx m_{2}$ ). Then, differential capital intensities in the two sectors combined with capital deepening in the economy (which itself results from technological progress) ensure faster growth in the more capital-intensive sector, sector 2. Intuitively, if capital were allocated proportionately to the two sectors, sector 2 would grow faster. Because of the changes in prices, capital and labor are reallocated in favor of the less capital-intensive sector, so that relative employment in sector 1 increases. However, crucially, this reallocation is not enough to fully offset the faster growth of real output in the more capital-intensive sector. This result also highlights that the assumption of balanced technological progress in Theorem 1 (which, in this context, corresponds to $m_{1}=m_{2}$ ) was not necessary for the result, but we simply needed to rule out the precise relative rate of technological progress between the two sectors that would ensure balanced growth (in this context, $m_{1} / \alpha_{1}=m_{2} / \alpha_{2}$ ).

Second, while the CGP growth rates look somewhat complicated because they are written in the general case, they are relatively simple once we restrict attention to parts of the parameter space. For instance, when $m_{1} / \alpha_{1}<m_{2} / \alpha_{2}$, the capital-intensive sector (sector 2 ) always grows faster than the labor-intensive one, i.e., $g_{1}^{*}<g_{2}^{*}$. In addition if $\varepsilon<1$, the more slowly-growing labor-intensive sector dominates the asymptotic behavior of the economy, and the CGP growth 
rates are

$$
\begin{aligned}
g^{*} & =g_{C}^{*}=g_{1}^{*}=z_{1}^{*}=n+\frac{1}{\alpha_{1}(\nu-1)} m_{1}, \\
g_{2}^{*} & =n+\frac{\varepsilon m_{2}}{(\nu-1)}+\frac{\left[1-\alpha_{2}\left(1-\varepsilon \alpha_{2}(1-\varepsilon)\right)\right] m_{1}}{\alpha_{1}(\nu-1)\left[1-\alpha_{2}(1-\varepsilon)\right]}>g^{*} .
\end{aligned}
$$

In contrast, when $\varepsilon>1$, the more rapidly-growing capital-intensive sector dominates the asymptotic behavior of the economy and

$$
\begin{aligned}
g^{*} & =g_{C}^{*}=g_{2}^{*}=z_{2}^{*}=n+\frac{1}{\alpha_{2}(\nu-1)} m_{2}, \\
g_{1}^{*} & =n+\frac{\varepsilon m_{1}}{(\nu-1)}+\frac{\left[1-\alpha_{1}\left(1-\varepsilon \alpha_{1}(1-\varepsilon)\right)\right] m_{2}}{\alpha_{2}(\nu-1)\left[1-\alpha_{1}(1-\varepsilon)\right]}<g^{*} .
\end{aligned}
$$

Third, as the proof of Theorem 3 makes it clear, in the limiting equilibrium the share of capital and labor allocated to one of the sector tends to one (e.g., when sector 1 is the asymptotically dominant sector, $\lambda^{*}=\kappa^{*}=1$ ). Nevertheless, at all points in time both sectors produce positive amounts, so this limit point is never reached. In fact, at all times both sectors grow at rates greater than the rate of population growth in the economy. Moreover, when $\varepsilon<1$, the sector that is shrinking grows faster than the rest of the economy at all point in time, even asymptotically. Therefore, the rate at which capital and labor are allocated away from this sector is determined in equilibrium to be exactly such that this sector still grows faster than the rest of the economy. This is the sense in which non-balanced growth is not a trivial outcome in this economy (with one of the sectors shutting down), but results from the positive but differential growth of the two sectors.

Finally, it can be verified that the share of capital in national income and the interest rate are constant in the CGP. For example, when $m_{1} / \alpha_{1}<m_{2} / \alpha_{2}, \sigma_{K}^{*}=1-\alpha_{1}$ and when $m_{1} / \alpha_{1}>$ $m_{2} / \alpha_{2}, \sigma_{K}^{*}=1-\alpha_{2}$-in other words, the asymptotic capital share in national income will reflect the capital share of the dominant sector. The limiting interest rate, on the other hand, will be equal to $r^{*}=\left(1-\alpha_{1}\right) \gamma^{\frac{\varepsilon}{\varepsilon-1}}\left(\chi^{*}\right)^{-\alpha_{1}}$ in the first case and $r^{*}=\left(1-\alpha_{2}\right) \gamma^{\frac{\varepsilon}{\varepsilon-1}}\left(\chi^{*}\right)^{-\alpha_{2}}$ in the second case, where $\chi^{*}$ is effective capital-labor ratio defined in equation (57) below. These results are the basis of the claim in the Introduction that this equilibrium may account for non-balanced growth at the sectoral level, without substantially deviating from the Kaldor facts. In particular, the constant growth path equilibrium matches both the Kaldor facts and generates unequal growth between the two sectors. However, in this constant growth path equilibrium, one of the sectors has already become very small relative to the other. 
Therefore, this theorem does not answer whether along the equilibrium path (but away from the asymptotic equilibrium), we can have a situation in which both sectors have non-trivial employment levels and the equilibrium capital share in national income and the interest rate are approximately constant. This question and the stability of the constant growth path equilibrium are investigated next.

\subsection{Dynamics and Stability}

The previous section characterized the asymptotic equilibrium, and established the existence of a unique constant growth path. This growth path exhibits non-balanced growth, though asymptotically the economy grows at a constant rate and the share of capital in national income is constant. We now study the equilibrium behavior of this economy away from this asymptotic equilibrium.

The equilibrium behavior away from the asymptotic equilibrium path can be represented by a dynamical system characterizing the behavior of a control variable $C$ and four state variables $K, L, M_{1}$ and $M_{2}$. The dynamics of aggregate consumption, $C$, and aggregate capital stock, $K$, are given by the Euler equation (43) and the resource constraint (16). Furthemore, the dynamic behavior of $L$ is given by (12) and those of $M_{1}$ and $M_{2}$ are given by (21).

As noted above, when $\varepsilon>1$, the sector which grows faster dominates the economy, while when $\varepsilon<1$, conversely, the slower sector dominates. We will show that in both cases the unique CGP of the previous section is locally stable. Without loss of generality, we restrict the discussion to the case in which asymptotically the economy is dominated by the labor-intensive sector, sector 1 , so that

$$
g^{*}=g_{1}^{*}=z_{1}^{*}=n+\frac{1}{\alpha_{1}(\nu-1)} m_{1} .
$$

This means that when we assume $\varepsilon<1$, the relevant part of the parameter space is where $m_{1} / \alpha_{1}<m_{2} / \alpha_{2}$, and, when $\varepsilon>1$, we must have $m_{1} / \alpha_{1}>m_{2} / \alpha_{2}$ (for the rest of the parameter space, it would be sector 2 that dominates the asymptotic behavior, and the same results apply analogously).

The equilibrium behavior of this economy can be represented by a system of autonomous non-linear differential equations in three variables,

$$
c \equiv \frac{C}{L M_{1}^{\frac{1}{\alpha_{1}(\nu-1)}}}, \chi \equiv \frac{K}{L M_{1}^{\frac{1}{\alpha_{1}(\nu-1)}}} \text { and } \kappa .
$$


Here $c$ is the level of consumption normalized by population and technology (of the sector that will dominate the asymptotic behavior) and is the only control variable; $\chi$ is the capital stock normalized by the same denominator, and $\kappa$ determines the allocation of capital between the two sectors. These two are state variables with given initial conditions $\chi(0)$ and $\kappa(0) .{ }^{16}$

The dynamic equilibrium conditions then translate into the following equations:

$$
\begin{aligned}
\frac{\dot{c}}{c} & =\frac{1}{\theta}\left[\left(1-\alpha_{1}\right) \gamma\left(\frac{Y}{Y_{1}}\right)^{\frac{1}{\varepsilon}} \lambda^{\alpha_{1}}(\kappa \chi)^{-\alpha_{1}}-\rho\right]-n-\frac{1}{\alpha_{1}(\nu-1)} m_{1}, \\
\frac{\dot{\chi}}{\chi} & =\lambda^{\alpha_{1}} \kappa^{1-\alpha_{1}} \chi^{-\alpha_{1}}\left(\frac{Y}{Y_{1}}\right)-\chi^{-1} c-n-\frac{1}{\alpha_{1}(\nu-1)} m_{1}, \\
\frac{\dot{\kappa}}{\kappa} & =\frac{(1-\kappa)\left[\left(\alpha_{1}-\alpha_{2}\right) \frac{\dot{\chi}}{\chi}+\left(\frac{1}{\nu-1}\right)\left(m_{2}-\frac{\alpha_{2}}{\alpha_{1}} m_{1}\right)\right]}{\left(\frac{\varepsilon}{1-\varepsilon}\right)+\alpha_{2}+\left(1-\alpha_{1}\right)(1-\kappa)+\left(1-\alpha_{2}\right) \kappa+\left(\alpha_{1}-\alpha_{2}\right)(1-\lambda)},
\end{aligned}
$$

where

$$
\left(\frac{Y}{Y_{1}}\right)=\gamma^{\frac{\varepsilon}{\varepsilon-1}}\left[1+\left(\frac{1-\alpha_{1}}{1-\alpha_{2}}\right)\left(\frac{1}{\kappa}-1\right)\right]^{\frac{\varepsilon}{\varepsilon-1}},
$$

and

$$
\lambda=\left[\left(\frac{1-\alpha_{1}}{\alpha_{1}}\right)\left(\frac{\alpha_{2}}{1-\alpha_{2}}\right)\left(\frac{1-\kappa}{\kappa}\right)+1\right]^{-1} .
$$

Clearly, the constant growth path equilibrium characterized above corresponds to a steadystate equilibrium in terms of these three variables, denoted by $c^{*}, \chi^{*}$ and $\kappa^{*}$ (i.e., in the CGP equilibrium, $c, \chi$ and $\kappa$ will be constant). These steady-state values are given by $\kappa^{*}=1$,

$$
\chi^{*}=\left[\frac{\left(\theta\left[n+\frac{1}{\alpha_{1}(\nu-1)} m_{1}\right]+\rho\right)}{\gamma^{\frac{\varepsilon}{\varepsilon-1}}\left(1-\alpha_{1}\right)}\right]^{-\frac{1}{\alpha_{1}}},
$$

and

$$
c^{*}=\gamma^{\frac{\varepsilon}{\varepsilon-1}} \chi^{*^{1-\eta}}-\chi^{*}\left(n+\frac{1}{\alpha_{1}(\nu-1)} m_{1}\right) .
$$

Since there are two state and one control variable, local (saddle-path) stability requires the existence of a (unique) two-dimensional manifold of solutions in the neighborhood of the steady state that converge to $c^{*}, \chi^{*}$ and $\kappa^{*}$. The next theorem states that this is the case.

Theorem 4 The non-linear system (58) is locally (saddle-path) stable, in the sense that in the neighborhood of $c^{*}, \chi^{*}$ and $\kappa^{*}$, there is a unique two-dimensional manifold of solutions that converge to $c^{*}, \chi^{*}$ and $\kappa^{*}$.

\footnotetext{
${ }^{16} \chi(0)$ is given by definition, and $\kappa(0)$ is uniquely pinned down by the static equilibrium allocation of capital at time $t=0$, given by (33).
} 
Proof. Let us rewrite the system (58) in a more compact form as

$$
\dot{x}=f(x),
$$

where $x \equiv\left(\begin{array}{lll}c & \chi & \kappa\end{array}\right)^{\prime}$. To investigate the dynamics of the system (59) in the neighborhood of the steady state, consider the linear system

$$
\dot{z}=J\left(x^{*}\right) z,
$$

where $z \equiv x-x^{*}$ and $x^{*}$ such that $f\left(x^{*}\right)=0$, where $J\left(x^{*}\right)$ is the Jacobian of $f(x)$ evaluated at $x^{*}$. Differentiation and some algebra enable us to write this Jacobian matrix as

$$
J\left(x^{*}\right)=\left[\begin{array}{ccc}
a_{c c} & a_{c \chi} & a_{c \kappa} \\
a_{\chi c} & a_{\chi \chi} & a_{\chi \kappa} \\
a_{\kappa c} & a_{\kappa \chi} & a_{\kappa \kappa}
\end{array}\right],
$$

where

$$
\begin{aligned}
& a_{c c}=a_{\kappa c}=a_{\kappa \chi}=0 \\
& a_{c \chi}=-\gamma^{\frac{\varepsilon}{\varepsilon-1}}\left(\chi^{*}\right)^{-\alpha_{1}-1}\left(\frac{\alpha_{1}\left(1-\alpha_{1}\right)}{\theta}\right) \\
& a_{c \kappa}=\gamma^{\frac{\varepsilon}{\varepsilon-1}}\left(\chi^{*}\right)^{-\alpha_{1}}\left(\frac{1-\alpha_{1}}{\theta}\right)\left[\left(\frac{1-\alpha_{1}}{1-\alpha_{2}}\right)\left(\frac{1+\alpha_{2}(1-\varepsilon)}{1-\varepsilon}\right)-\alpha_{1}\right] \\
& a_{\chi c}=-\left(\chi^{*}\right)^{-1} \\
& a_{\chi \chi}=\gamma^{\frac{\varepsilon}{\varepsilon-1}}\left(\chi^{*}\right)^{-\alpha_{1}-1}\left(1-\alpha_{1}\right)\left[1-\frac{1}{\theta}\right]+\frac{\left(\chi^{*}\right)^{-1} \rho}{\theta} \\
& a_{\chi \kappa}=\gamma^{\frac{\varepsilon}{\varepsilon-1}}\left(\chi^{*}\right)^{-\alpha_{1}}\left[\left(1-\alpha_{1}\right)+\left(\frac{1-\alpha_{1}}{1-\alpha_{2}}\right)\left(\frac{1+\alpha_{2}(1-\varepsilon)}{1-\varepsilon}\right)\right] \\
& a_{\kappa \kappa}=-\left(\frac{1-\varepsilon}{\nu-1}\right)\left(m_{2}-\frac{\alpha_{2}}{\alpha_{1}} m_{1}\right) .
\end{aligned}
$$

The determinant of the Jacobian is $\operatorname{det}\left(J\left(x^{*}\right)\right)=-a_{\kappa \kappa} a_{c \chi} a_{\chi c}$. The above expressions show that $a_{c \chi}$ and $a_{\chi c}$ are negative. Next, it can be seen that $a_{\kappa \kappa}$ is always negative since $\varepsilon \lessgtr 1 \Leftrightarrow$ $m_{2} / \alpha_{2} \gtrless m_{1} / \alpha_{1}$. [As noted above, this is not a parameter restriction. For example, when $\varepsilon>1$ and $m_{2} / \alpha_{2}>m_{1} / \alpha_{1}$, it will be sector 2 that grows more slowly in the limit, and stability will again obtain]. The fact that $a_{\kappa \kappa}<0$ immediately implies that $\operatorname{det}\left(J\left(x^{*}\right)\right)>0$, so the steady state is hyperbolic. Moreover, either all the eigenvalues are positive or two of them are negative and one is positive. To determine which is the case, we look at the characteristic equation given by $\operatorname{det}\left(J\left(x^{*}\right)-\mathbf{v} I\right)=0$, where $\mathbf{v}$ denotes the vector of the eigenvalues. This equation can be expressed as the following cubic in $v$, with roots corresponding to the eigenvalues:

$$
\left(a_{\kappa \kappa}-v\right)\left[v\left(a_{\chi \chi}-v\right)+a_{\chi c} a_{c \chi}\right]=0 .
$$


This expression shows that one of the eigenvalue is equal to $a_{\kappa \kappa}$ and thus negative, so there must be two negative eigenvalues. This establishes the existence of a unique two-dimensional manifold of solutions in the neighborhood of this steady state, converging to it. This proves local (saddle-path) stability.

This result shows that the constant growth path equilibrium is locally stable, and when the initial values of capital, labor and technology are not too far from the constant growth path, the economy will indeed converge to this equilibrium, with non-balanced growth at the sectoral level and constant capital share and interest rate at the aggregate.

\section{A Simple Calibration}

We now undertake an illustrative calibration of the model presented in Section 3 to investigate whether the equilibrium dynamics generated by our model economy are broadly consistent with non-balanced growth at the sectoral level and the aggregate Kaldor facts in the US data. To do this, we use by-industry data on value added and employment and national data on capital from NIPA as described in Appendix B. To map our model economy to data, we classify the NIPA two-digit (22-level) industries into low and high capital intensity "sectors", each comprising approximately $50 \%$ of total employment. High capital intensity sectors have capital share in value added of greater than 0.35. Appendix B and Table B1 provide more details on the classification of the sectors. This division of sectors into high and low capital intensity sectors is also the one used in Figure 2. We use this division of sectors together with other aggregate data from NIPA for the calibration of our benchmark model.

It can be verified that in our benchmark model $\gamma, M_{1}$ and $M_{2}$ do not matter separately.

Instead, all expressions are in terms of $B_{1} \equiv \gamma^{\frac{\varepsilon}{\varepsilon-1}} M_{1}^{\frac{1}{\nu-1}}$ and $B_{2} \equiv(1-\gamma)^{\frac{\varepsilon}{\varepsilon-1}} M_{2}^{\frac{1}{\nu-1}}$. Consequently, our model economy is fully characterized by eight parameters, $\varepsilon, \alpha_{1}, \alpha_{2}, \rho, \theta, n$, $\hat{m}_{1} \equiv m_{1} /(\nu-1)$ and $\hat{m}_{2} \equiv m_{1} /(\nu-1)$, and six initial values, $L(0), K(0), B_{1}(0), B_{2}(0)$, $Y_{1}^{N}(0)$ and $Y_{2}^{N}(0)$, where $Y_{j}^{N}$ 's refer to nominal output levels (thus in terms of our model, $\left.Y_{j}^{N}(0)=p_{j}(0) Y_{j}(0)\right)$.

We choose a period to correspond to a year and take the initial year, $t=0$, to correspond to the first year for which we have NIPA data for our sectors, 1948. We adopt the following standard parameter values:

- $1 \%$ annual population growth, $n=0.01$ 
- $2 \%$ annual discount rate, $\rho=0.02$;

- $10 \%$ annual asymptotic "rental rate" of capital, $r^{*}=0.10$;

- $2 \%$ annual asymptotic growth rate, $g^{*}=0.02 ;^{17}$

Our classification of industries creates two sectors, with corresponding shares of labor in value added of 0.72 and 0.52 . Consequently we take $\alpha_{1}=0.72$ and $\alpha_{2}=0.52$ (see Appendix B). For our benchmark calibration we set $\hat{m}_{1}=\hat{m}_{2}$, so that the asymptotically dominant sector will be the less capital-intensive sector, sector 1 . In this case, our model economy implies that the asymptotic growth rate of aggregate output satisfies

$$
g^{*}=g_{1}^{*}=n+\hat{m}_{1} / \alpha_{1},
$$

which, together with the values for $g^{*}, n$ and $\alpha_{1}$, implies $\hat{m}_{1}=\hat{m}_{2}=0.0072$. We will report robustness checks for different levels of $\hat{m}_{2}$ below (while keeping sector 1 the asymptotically dominant sector). The general patterns implied by our model are not sensitive to the exact value of $\hat{m}_{2}$. Note also that, since sector 1 is the asymptotically dominant sector, our model pins down the asymptotic share of capital in national income as $\sigma_{K}^{*}=1-\alpha_{1}=0.28$, which is lower than the share of capital in the US data shown in Figure 1.

Moreover, since the growth rate of consumption and output have to be equal asymptotically, the Euler equation (43) implies that $g^{*}=\left(r^{*}-\rho\right) / \theta$, thus $\theta=4$, which results in a reasonable elasticity of intertemporal substitution of 0.25 .

From NIPA, we take the following data for 1948: $L(0)=37,169, K(0)=560,000$, $Y_{1}^{N}(0)=85,885, Y_{2}^{N}(0)=108,473, Y^{N}(0)=194,358$, where $Y^{N}(0)$ refers to total nominal output in all the sectors under consideration. Employment is in terms of full-time equivalents in thousands, while all the other figures are in terms of (current) millions of dollars (see Appendix B). These numbers will be used to determine the initial values for our calibration.

Next, notice that equation (33) can be rewritten as:

$$
\kappa=\left[1+\left(\frac{1-\alpha_{2}}{1-\alpha_{1}}\right) \frac{Y_{2}^{N}}{Y_{1}^{N}}\right]^{-1} .
$$

\footnotetext{
${ }^{17}$ These numbers are the same as those used by Barro and Sala-i-Martin (2004) in their calibration of the baseline neoclassical model. The only difference is that since there is no depreciation in our model, we choose $r$ as $10 \%$ to correspond to the rental rate of capital inclusive of depreciation.
} 
This equation together with the 1948 levels of nominal output in the two sectors, $Y_{1}^{N}(0)$ and $Y_{2}^{N}(0)$, from NIPA pins down $\kappa(0)$. Equation (34) then gives the initial value of relative employment, $\lambda(0)$. These implied values are:

$$
\lambda(0)=0.52 \text { and } \kappa(0)=0.32 .
$$

We can also compute an empirical equivalent of $\lambda(0)$ from NIPA data using employment in different industries. Reassuringly, this empirical counterpart to $\lambda(0)$ is approximately 0.46 , which is reasonably close to the number implied by our model. Moreover, these numbers also pin down the initial interest rate and the capital share in national income as $r(0)=0.14$ and $\sigma_{K}(0)=0.39 .{ }^{18}$ Recall that we chose the asymptotic interest rate as $r^{*}=0.10$ and our model economy implies that the limiting capital share is $\sigma_{K}^{*}=0.28$, thus both the interest rate and the capital share must decline at some point along the transition path. A key question concerns the speed of these declines.

We also choose $\varepsilon=0.5$ as our benchmark value, which is consistent with the evidence discussed in footnote 5. We will experiment with different values of $\varepsilon$ and show that the precise value of the elasticity of substitution does not matter for the patterns documented in our calibration.

This only leaves the initial values of $B_{1}(0)$ and $B_{2}(0)$ to be determined. We do this using equations (22) and (28) combined with the data from NIPA for $Y_{1}^{N}(0), Y_{2}^{N}(0)$ and $Y^{N}(0)$ introduced above. In particular, with the transformed variables, equation (22) can be rewritten as:

$$
\frac{Y_{1}^{N}(0)}{Y_{2}^{N}(0)}=\left(\frac{B_{1}(0)\left(K_{1}(0)\right)^{1-\alpha_{1}}\left(L_{1}(0)\right)^{\alpha_{1}}}{B_{2}(0)\left(K_{2}(0)\right)^{1-\alpha_{2}}\left(L_{2}(0)\right)^{\alpha_{2}}}\right)^{\frac{\varepsilon-1}{\varepsilon}}
$$

This equation combined with the production function from (28) yields the initial values $B_{1}(0)$ and $B_{2}(0)$ necessary for our calibration exercise.

Figure 3 shows the results of the benchmark calibration. ${ }^{19}$ The four panels depict relative employment in sector $1(\lambda)$, relative capital in sector $1(\kappa)$, the interest rate $(r)$ and the

\footnotetext{
${ }^{18}$ For the capital share, we use equation (89) in Appendix B. For the interest rate, we use equation (31) or (32), together with the aggregate capital stock of the economy.

${ }^{19}$ To compute the dynamics of our calibrated economy, we first represent the equilibrium as a two-dimensional non-autonomous system in $c$ and $\chi$ (rather than the three-dimensional autonomous system analyzed above) since $\kappa$ can be represented as a function of time only. This two dimensional system has one state and one control variable. Following Judd (1998, chapter 10), we then discretize these differential equations using the Euler method, and turn them into a system of first-order difference equations in $c_{t}$ and $\chi_{t}$, which can itself be transformed into a second-order non-autonomous system only in $\chi_{t}$. We compute a numerical solution to the
} 
capital share in national income $\left(\sigma_{K}\right)$. The solid line is for the benchmark economy, with $\hat{m}_{1}=\hat{m}_{2}=0.0072$ and $\varepsilon=0.5$. The other two lines show the results of the calibration for $\varepsilon=0.4$ and $\varepsilon=0.6$ (while keeping $\hat{m}_{2}=0.0072$ ).
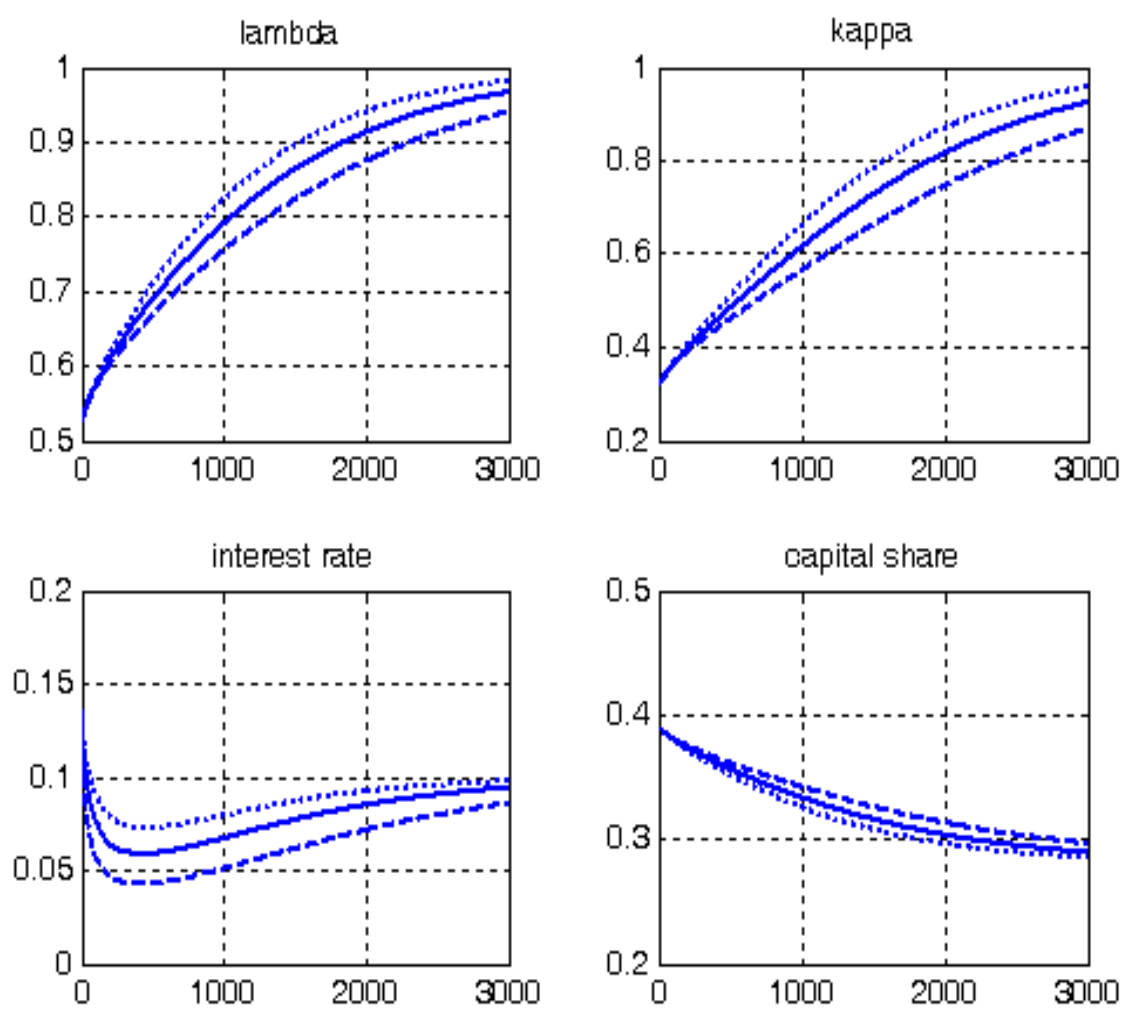

Figure 3: Behavior of $\kappa, \lambda, r$ and $\sigma_{K}$ in the model economy. Solid line: benchmark model, $\hat{m}_{2}=0.0072, \varepsilon=0.5$. Dotted line: $\hat{m}_{2}=0.0072, \varepsilon=0.4$. Dashed line: $\hat{m}_{2}=0.0072, \varepsilon=0.6$. See text for further details.

Let us first focus on the benchmark simulation, given by the solid line in Figure 3. The first noteworthy feature is the rate of convergence. The units on the horizontal axis are years and range from 0 to 3000 . This shows that it takes a very long time for the fraction of capital and labor allocated to sector 1 to approach their asymptotic equilibrium value of 1 . For example, second-order difference equation by choosing a polynomial form for the endogenous variables:

$$
\left(c_{t}, \kappa_{t}, \chi_{t}\right)=F(t ; \theta) .
$$

We find the vector $\theta$ that minimizes the squared residuals of the difference equations, using non-linear least squares (see, e.g., Judd, 1998, chapter 4). 
initially, about $52 \%$ of employment is in sector 1, and after 500 years, this number has increased only to about $70 \%$. The implied allocation of capital between the two sectors is a similar. This illustrates that even though in the limiting equilibrium one of the sectors employs all of the factors, it takes a very long time for the economy to approach this limit point. Second, the rental rate of capital (or the interest rate) follows a non-monotonic pattern, but does not vary by much (remaining between $6 \%$ and $10 \%$ for most of the 3000 years). Third, the share of capital in national income is declining visibly. As noted above, this is by construction, since the initial capital share is set as $\sigma_{K}(0)=0.39$, while the model implies that the asymptotic capital share is $\sigma_{K}^{*}=0.28$. More importantly, the decline of the capital share is relatively slow.

These patterns can be seen most clearly in Table 1, which shows US data from 1948 to 2004 for the relative (real) output and employment of the high and low capital intensity sectors and the capital share in national income (see Appendix B) as well as the results from the calibration of the model for the same time period. In particular, since $t=0$ for the calibration is taken to be 1948, the implied numbers for 2004 are given by the calibration for the time period $t=56$. The first two columns of this table confirm the pattern shown in Figure 2 in the Introduction; real output grows faster in high capital intensity sectors, while employment grows faster in low capital intensity sectors. The next two columns show that the benchmark calibration is consistent with this pattern, though the magnitude of changes is somewhat smaller in the calibration than in the data. For example, $Y_{2} / Y_{1}$ increases by about $25 \%$ in the data between 1948 and 2004, while the increase is about $15 \%$ in the benchmark calibration. Similarly, $L_{2} / L_{1}$ declines by about $33 \%$ in the data, while the implied decline in the model is about $12 \% .{ }^{20}$ The movements of the capital share in national income, on the other hand, are similar in both cases. In the data there is a decline from 0.41 to 0.39 between 1948 and 2004, whereas in the model there is a slightly smaller decline from 0.39 to 0.38 . These results suggest that our model economy generates changes in the relative output and employment of the two sectors that are quantitatively as well as qualitatively plausible, even though the magnitudes of the changes are about half as large as those in the data.

\footnotetext{
${ }^{20}$ Note that the initial values of $Y_{2} / Y_{1}$ and $L_{2} / L_{1}$ (and $\lambda$ ) are not the same in the data and in the benchmark model, since, as explained above, we chose the sectoral allocation of labor implied by the model given the relative nominal outputs of the two sectors (recall equation (60)).
} 
Table 1: Data and Model Calibration, 1948-2004

\begin{tabular}{|l|c|c|c|c|c|c|c|c|}
\hline \hline & \multicolumn{2}{|c|}{ US Data } & \multicolumn{2}{c|}{$\begin{array}{c}\text { Benchmark Model } \\
\varepsilon=0.5, \hat{m}_{2}=0.0072\end{array}$} & \multicolumn{2}{c|}{$\begin{array}{c}\text { Model } \\
\end{array}$} & & \multicolumn{2}{|c|}{$\varepsilon=0.4, \hat{m}_{2}=0.0072$} & \multicolumn{2}{c|}{ Model } \\
& 1948 & 2004 & 1948 & 2004 & 1948 & 2004 & 1948 & 2004 \\
\hline \hline & 0.85 & 1.06 & 0.79 & 0.91 & 0.86 & 0.94 & 0.70 & 0.87 \\
\hline \hline$Y_{2} / Y_{1}$ & 1.16 & 0.77 & 0.91 & 0.80 & 0.91 & 0.80 & 0.91 & 0.79 \\
$L_{2} / L_{1}$ & 0.41 & 0.39 & 0.39 & 0.38 & 0.39 & 0.38 & 0.39 & 0.38 \\
$\sigma_{k}$ & 0.49 \\
\hline \hline
\end{tabular}

Note: The data are from NIPA (see Appendix B). The model calibrations are described in the text.

The other columns of the table show that the results are broadly similar for other values of the elasticity of substitution, $\varepsilon$. When $\varepsilon$ is smaller than the benchmark value of $\varepsilon=0.5$, the change in relative employment levels is similar to the benchmark, and the change in relative output levels is somewhat smaller. Interestingly, when $\varepsilon$ is larger, both employment and output movements are larger and more comparable to the data. For example, Table 1 shows that for $\varepsilon=0.6$, the implied increase in $Y_{2} / Y_{1}$ between 1948 and 2004 is $25 \%$ as in the data.

Figure 4 and Table 2 show the results of the calibration for different values of $\hat{m}_{2}$, again together with the benchmark for comparison. The broad patterns implied by different values of $\hat{m}_{2}$ are again similar to the benchmark calibration. When $\hat{m}_{2}$ is taken to be smaller (0.0062), the changes in $Y_{2} / Y_{1}$ and $L_{2} / L_{1}$ are somewhat less pronounced; in contrast when $\hat{m}_{2}$ is taken to be 0.0082 , there are greater relative employment and relative output changes. For example, with $\hat{m}_{2}=0.0082, Y_{2} / Y_{1}$ increases by about $28 \%$, while $L_{2} / L_{1}$ declines by almost $15 \%$.

Table 2: Data and Model Calibration, 1948-2004 (continued)

\begin{tabular}{|l|c|l|c|c|c|c|c|c|}
\hline \hline & \multicolumn{2}{|c|}{ US Data } & \multicolumn{2}{c|}{$\begin{array}{c}\text { Benchmark Model } \\
\varepsilon=0.5, \hat{m}_{2}=0.0072\end{array}$} & \multicolumn{2}{c|}{ Model } & \multicolumn{2}{c|}{ Model } \\
& & & \multicolumn{2}{|c|}{$\hat{m}_{2}=0.0062$} & \multicolumn{2}{c|}{$\varepsilon=0.5, \hat{m}_{2}=0.0082$} \\
\hline \hline & 1948 & 2004 & 1948 & 2004 & 1948 & 2004 & 1948 & 2004 \\
\hline \hline$Y_{2} / Y_{1}$ & 0.85 & 1.06 & 0.79 & 0.91 & 0.70 & 0.84 & 0.70 & 0.90 \\
$L_{2} / L_{1}$ & 1.16 & 0.77 & 0.91 & 0.80 & 0.91 & 0.81 & 0.91 & 0.78 \\
$\sigma_{k}$ & 0.41 & 0.39 & 0.39 & 0.38 & 0.39 & 0.39 & 0.39 & 0.38 \\
\hline \hline
\end{tabular}

Note: The data are from NIPA (see Appendix B). The model calibrations are described in the text.

We have also experimented with different values of $\varepsilon$ and $\hat{m}_{2}$ (subject to the constraint that $\hat{m}_{2} / \alpha_{2}>\hat{m}_{1} / \alpha_{1}$ ) and obtained similar results. Moreover, the results of the calibration are very similar if we use the initial value of $\lambda(0)$ implied by the sectoral employment data 

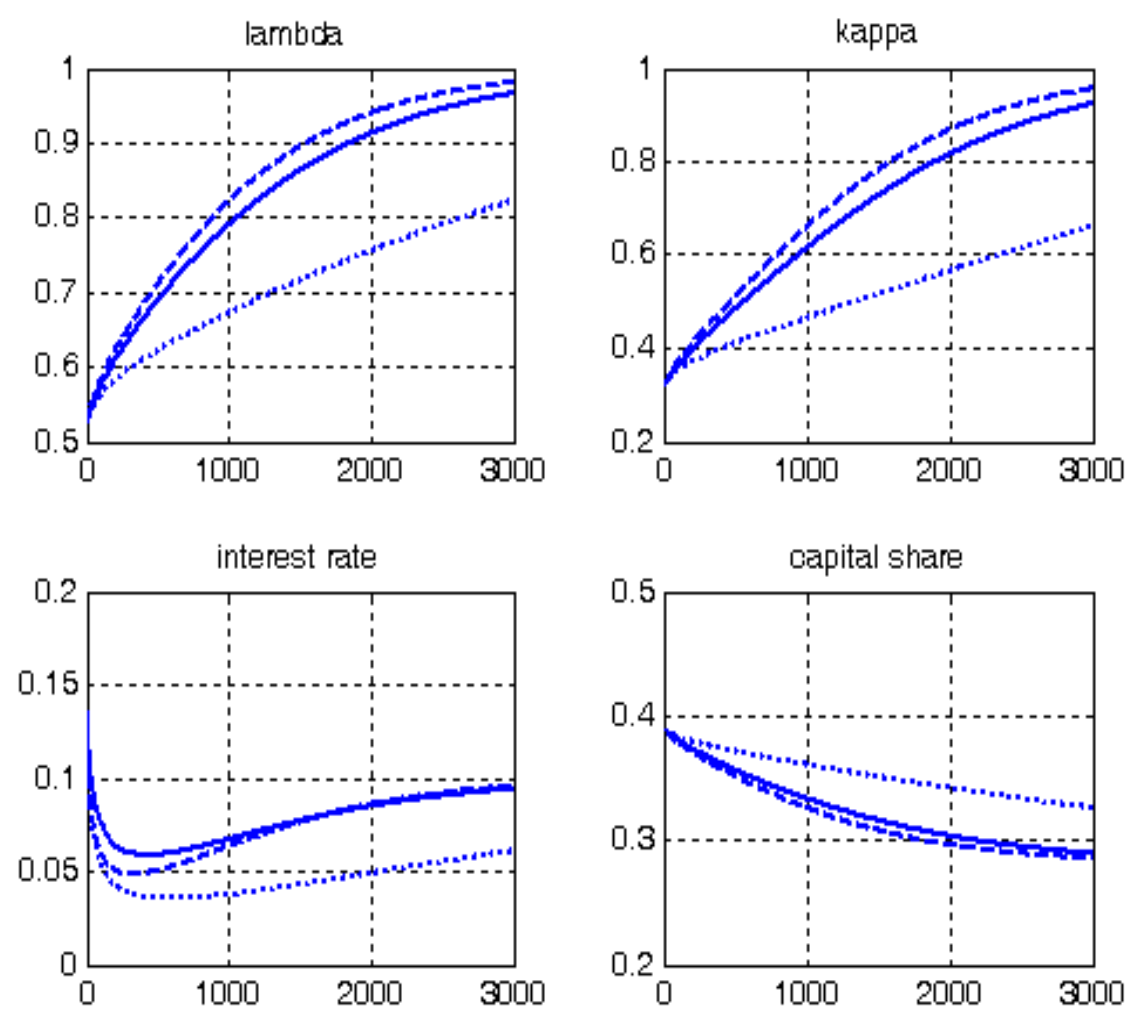

Figure 4: Behavior of $\kappa, \lambda, r$ and $\sigma_{K}$ in the model economy. Solid line: benchmark model, $\hat{m}_{2}=0.0072, \varepsilon=0.5$. Dotted line: $\hat{m}_{2}=0.0062, \varepsilon=0.5$. Dashed line: $\hat{m}_{2}=0.0082, \varepsilon=0.5$. See text for further details.

rather than the value implied by equation (60) above and the ratio of nominal output levels from NIPA (i.e., $\lambda(0)$ equal to 0.46 instead of 0.52 ). These details are available upon request.

Overall, we interpret the findings in this section as indicating that the mechanism proposed in this paper is capable of generating changes in the sectoral composition of employment and output that are broadly comparable with, though about half of the magnitude as, the changes we observe in the US data. Since the model does not include non-homothetic preferences, the fact that the magnitudes are somewhat smaller than those in the data is in fact reassuring and suggests that a more complete model incorporating both the mechanism proposed in this paper and non-homothetic preferences is worth investigating theoretically and quantitatively. 


\section{Non-Balanced Endogenous Growth}

In this section we introduce endogenous technological progress. This investigation has two objectives. As already emphasized, non-balanced growth results from capital deepening, and so far, capital deepening was a consequence of exogenous technological change. The first question is whether endogenous technological change might restore balanced growth. The analysis in this section will show that this is not the case. Second, endogenizing technological change in this context enables us to derive a model of non-balanced endogenous technological change, which is an important direction for models of endogenous technology, given the non-balanced nature of growth in the data. In particular, we will see that differences in capital intensity between sectors naturally lead to non-balanced endogenous technological progress.

Demographics, preferences and technology are the same as described in Section 3, with two differences. First, instead of the exogenous processes for technology given in (21), we now need to specify an innovation possibilities frontier, i.e., the technology to transform resources into blueprints for new varieties in the two sectors. We assume that

$$
\dot{M}_{1}=b_{1} M_{1}^{-\varphi} X_{1} \text { and } \dot{M}_{2}=b_{2} M_{2}^{-\varphi} X_{2}
$$

where $X_{1} \geq 0$ and $X_{2} \geq 0$ are research expenditures in terms of the final good, $b_{1}$ and $b_{2}$ are strictly positive constants measuring the technical difficulty of creating new blueprints in the two sectors, and $\varphi \in(-1, \infty)$ measures the degree of spillovers in technology creation. ${ }^{21}$

Second, we assume that firms that invent blueprints for intermediates in either sector become the monopolist supplier of that intermediate, with a fully-enforced perpetual patent. There is free entry into research, which means that any firm can make use of the innovation possibilities frontier (61) in order to invent new varieties.

The value of the monopolist for intermediate $i$ in sector $s$ is given by

$$
V_{s}(i, t)=\int_{t}^{\infty} \exp \left[-\int_{t}^{v} r(z) d z\right] \pi_{s}(i, v) d v
$$

\footnotetext{
${ }^{21}$ When $\varphi=0$, there are no spillovers from the current stock of knowledge to future innovations. With $\varphi<0$, there are positive spillovers and the stock of knowledge in a particular sector makes further innovation in that sector easier. With $\varphi>0$, there are negative spillovers ("fishing out") and further innovations are more difficult in sectors that are more advanced (see, for example, Jones, 1995, Kortum, 1997). Similar to the results in Jones (1995), Young (1999) and Howitt (1999), there will be endogenous growth for a range of values of $\varphi$ because of population growth. In the remainder, we will typically think of $\varphi>0$, so that there are negative spillovers, though this is not important for any of the asymptotic results.

Also, this innovation possibilities frontier assumes that only the final good is used to generate new technologies. The alternative is to have a scarce factor, such as labor or scientists, in which case some amount of positive spillovers would be necessary to generate endogenous growth.
} 
for $s=1,2$, where $\pi_{s}(i, t)=\left(q_{s}(i, t)-m c_{s}(i, t)\right) y_{s}(i, t)$ is the flow profits for firm $i$ at time $t$, with $q_{s}$ given by the iso-elastic demand curves in (23) in Section 3, and $m c_{s}$ is the marginal cost of production in this sector, which was also defined in Section 3. In equilibrium, all firms in the same sector will make the same profits, so $V_{s}(i, t)=V_{s}(t)$, and we use $V_{1}(t)$ and $V_{2}(t)$ to denote the values of firms in the two sectors at time $t$.

Each monopolist chooses its price to maximize (62). Since demands are iso-elastic and prices at time $t$ only influence revenues and costs at that point, profit-maximizing prices will be given by a constant markup over marginal cost:

$$
\begin{aligned}
& q_{1}(i)=\left(\frac{\nu}{\nu-1}\right) \alpha_{1}\left(1-\alpha_{1}\right)^{\alpha_{1}-1} r^{1-\alpha_{1}} w^{\alpha_{1}}, \\
& q_{2}(i)=\left(\frac{\nu}{\nu-1}\right) \alpha_{2}\left(1-\alpha_{2}\right)^{\alpha_{2}-1} r^{1-\alpha_{2}} w^{\alpha_{2}} .
\end{aligned}
$$

These prices are identical to the intermediate prices in Section 3, (24) and (25), except for the presence of the markup terms, $\nu /(\nu-1)$. Now, combining equations $(22),(23),(26)$ and (27) from Section 3 (which all still apply in the endogenous growth economy), the prices for the labor- and capital-intensive intermediates can be expressed as $q_{1}=\gamma M_{1}^{\frac{1}{\nu-1}}\left(Y_{1} / Y\right)^{-\frac{1}{\varepsilon}}$ and $q_{2}=(1-\gamma) M_{2}^{\frac{1}{\nu-1}}\left(Y_{2} / Y\right)^{-\frac{1}{\varepsilon}}$. These prices combined again with (26) and (27) and the fact that there is a markup of $\nu /(\nu-1)$ over marginal costs enable us to write the flow profits from the sale of the labor- and capital-intensive intermediates as:

$$
\pi_{1}=\frac{\gamma}{\nu}\left(\frac{Y_{1}}{Y}\right)^{-\frac{1}{\varepsilon}} \frac{Y_{1}}{M_{1}} \text { and } \pi_{2}=\frac{1-\gamma}{\nu}\left(\frac{Y_{2}}{Y}\right)^{-\frac{1}{\varepsilon}} \frac{Y_{2}}{M_{2}} .
$$

Because of the markups, factor prices are also slightly different in this section than in Section 3. In particular, we have:

$$
\begin{gathered}
w=\left(\frac{\nu-1}{\nu}\right) \gamma \alpha_{1}\left(\frac{Y}{Y_{1}}\right)^{\frac{1}{\varepsilon}} \frac{Y_{1}}{L_{1}} \text { and } w=\left(\frac{\nu-1}{\nu}\right)(1-\gamma) \alpha_{2}\left(\frac{Y}{Y_{2}}\right)^{\frac{1}{\varepsilon}} \frac{Y_{2}}{L_{2}}, \\
r=\left(\frac{\nu-1}{\nu}\right) \gamma\left(1-\alpha_{1}\right)\left(\frac{Y}{Y_{1}}\right)^{\frac{1}{\varepsilon}} \frac{Y_{1}}{K_{1}} \text { and } r=\left(\frac{\nu-1}{\nu}\right)(1-\gamma)\left(1-\alpha_{2}\right)\left(\frac{Y}{Y_{2}}\right)^{\frac{1}{\varepsilon}} \frac{Y_{2}}{K_{2}} .
\end{gathered}
$$

Even though factor prices include the markup term $(\nu-1) / \nu$, this markup is the same in both sectors. Consequently, the allocation of capital in labor between the two sectors is unchanged and is still determined by equations (33) and (34) in Section 3.

Given the value for being the monopolist for intermediate in (62), the free entry conditions take the following form:

$$
V_{1} \leq \frac{M_{1}^{\varphi}}{b_{1}} \text { and } V_{2} \leq \frac{M_{2}^{\varphi}}{b_{2}}
$$


with each condition holding as equality when there is positive R\&D expenditure for that sector, i.e., when $X_{1}>0$ or $X_{2}>0$.

The resource constraint of the economy is also modified to incorporate the R\&D expenditures becomes

$$
\dot{K}+C+X_{1}+X_{2} \leq Y
$$

Finally, the problem of consumer optimization is similar to that in Section 3. In particular, the Euler equation is still given by (43), but the transversality condition needs to be modified because of corporate profits. The financial wealth of the representative consumer now comes from payments to capital and profits and is thus given by $W(t)=K(t)+M_{1}(t) V_{1}(t)+$ $M_{2}(t) V_{2}(t)$, where recall that $V_{1}(t)$ is the present discounted value of the profits of a firm in the sector 1 at time $t$ and there are $M_{1}(t)$ such firms, and similarly for $V_{2}(t)$ and $M_{2}(t)$. The relevant transversality condition can then be written as

$$
\lim _{t \rightarrow \infty} W(t) \exp \left(-\int_{0}^{t} r(\tau) d \tau\right)=0
$$

Definition 5 A dynamic equilibrium is given by paths of wages and interest rates, $w$ and $r$, labor and capital allocation decisions, $\lambda$ and $\kappa$, satisfying (33), (34), (66) and (67), and of consumption $C$, employment, $L$, capital stock, $K$, technology, $M_{1}$ and $M_{2}$ values of innovation, $V_{1}$ and $V_{2}$, and research expenditures, $X_{1}$ and $X_{2}$, satisfying (12), (43), (61), (62), (68), (69) and (70).

It is also useful to define a path that satisfies all of these equations, possibly except the transversality condition, (70), as a quasi-equilibrium.

Since the case with $\varepsilon<1$ is both more interesting, and in view of the discussion in footnote 5 , also more realistic, in this section we focus on this case exclusively.

We first note that Propositions 1 and 2 from Section 3 still apply and characterize the comparative static responses. Moreover, Lemmas 1 and 2 from Section 3 continue to determine the behavior of the growth rate of sectoral output, capital and employment. For the analysis of the economy with endogenous technology, we also need an additional result in the next lemma. It shows that provided that (i) $\varepsilon<1$, (ii) there exists a constant asymptotic interest rate $r^{*}$ (i.e., $\lim _{t \rightarrow \infty} \dot{r}=0$ ), and (iii) there is positive population growth, in the asymptotic equilibrium the free entry conditions in (68) will both hold as equality: 
Lemma 3 Suppose that $\varepsilon<1, n>0$, and $\lim _{t \rightarrow \infty} \dot{r}=0$, then $\lim _{t \rightarrow \infty}\left(V_{1}-M_{1}^{\varphi} / b_{1}\right)=0$ and $\lim _{t \rightarrow \infty}\left(V_{2}-M_{2}^{\varphi} / b_{2}\right)=0$.

\section{Proof. See Appendix A.}

This lemma is an important result for the analysis of non-balanced endogenous growth. It implies that both free-entry conditions must hold as equalities in the limiting equilibrium and thus enables us to solve for the asymptotic growth rates from the free entry conditions and obtain a relatively simple characterization of the constant growth path equilibrium.

The economic intuition for the lemma comes from population growth; with population growth, it is always optimal to allocate more capital to each sector, which increases the profitability of intermediate producers in that sector. Consequently, the value of a new blueprint increases asymptotically. This rules out asymptotic equilibrium paths with slack free-entry conditions, because along such paths, the cost of creating a new blueprint would remain constant, ultimately violating the free-entry condition.

To establish the existence of a CGP, we now impose the following parameter restriction, which replaces (A2) in the exogenous technology case:

$$
\alpha_{1} \zeta>1 \text { and } \rho>(1-\theta) \frac{\alpha_{1} \zeta}{\alpha_{1} \zeta-1} n
$$

where $\zeta \equiv(\nu-1)(1+\varphi)$. Assumption A3 ensures that the transversality condition (70) holds. Notice that in contrast to Assumption A2 only $\alpha_{1}$ features in this condition; this is because Assumption A1 already imposed that $\alpha_{1}<\alpha_{2}$, and with endogenous technology, this is sufficient to make sector 1 the more slowly growing sector in the asymptotic equilibrium. Assumption A3 also rules out quasi-equilibrium paths where output and consumption grow more than exponentially. This is stated in the next lemma.

Lemma 4 Suppose Assumption A3 holds and $\varepsilon<1$, then there exists no quasi-equilibria with $\lim _{t \rightarrow \infty} \dot{C} / C=\infty$.

This lemma is also proved in Appendix A, where, for completeness, we also show that Assumption A3 is "tight" in the sense that, if first inequality in this assumption did not hold, there always exist quasi-equilibria with more than exponential growth (which would also naturally violate the transversality condition). The proof of this lemma also makes clear that the second part of Assumption A3 is also necessary for the transversality condition. 
Lemmas 3 and 4 together imply that the asymptotic equilibrium has to converge either to a limit with constant growth of consumption, or to a limit cycle. Using these results, the next theorem establishes the existence of a unique constant growth path, with non-balanced sectoral growth, but constant share of capital and constant interest rate in the aggregate. This theorem is therefore the analogue of Theorem 3 in Section 3.

Theorem 5 Suppose Assumptions A1 and A3 hold, $\varepsilon<1$, and $n>0$, then there exists a unique CGP where

$$
\begin{gathered}
g^{*}=g_{C}^{*}=g_{1}^{*}=z_{1}^{*}=\frac{\alpha_{1} \zeta}{\alpha_{1} \zeta-1} n, \\
g_{2}^{*}=\frac{\alpha_{1} \zeta(1-\varepsilon+\zeta)+\varepsilon \zeta\left(\alpha_{1}-\alpha_{2}\right)}{\left(\alpha_{1} \zeta-1\right)(1-\varepsilon+\zeta)} n>g^{*}, \\
z_{2}^{*}=\frac{\alpha_{1} \zeta(1-\varepsilon+\zeta)-\zeta\left(\alpha_{1}-\alpha_{2}\right)(1-\varepsilon)}{\left(\alpha_{1} \zeta-1\right)(1-\varepsilon+\zeta)} n<g^{*}, \\
n_{1}^{*}=n \quad \text { and } \quad n_{2}^{*}=\left[1-\frac{\zeta(1-\varepsilon)\left(\alpha_{1}-\alpha_{2}\right)}{\left(\alpha_{1} \zeta-1\right)[1-\varepsilon+\zeta]}\right] n, \\
m_{1}^{*}=\frac{1}{1+\varphi} z_{1}^{*} \quad \text { and } \quad m_{2}^{*}=\frac{1}{1+\varphi} z_{2}^{*} .
\end{gathered}
$$

\section{Proof. See Appendix A.}

A number of features are worth noting. First, this theorem shows that the equilibrium of this non-balanced endogenous growth economy takes a relatively simple form. Second, given the equilibrium rates of technological change in the two sectors, $m_{1}^{*}$ and $m_{2}^{*}$, the asymptotic growth rates are identical to those in Theorem 3 in Section 3, so that the economy with endogenous technological change gives the same insights as the one with exogenous technology. In particular, there is non-balanced sectoral growth, but in the aggregate, the economy has a limiting equilibrium with constant share of capital in national income and a constant interest rate.

Finally and interestingly, technology is also endogenously non-balanced, and in fact, tries to counteract the non-balanced nature of economic growth. Specifically, there is more technological change towards the sector that is growing more slowly (recall we are focusing on the case where $\varepsilon<1$ ), so that the sector with lower capital intensity employs an increasing share of the capital and labor in the economy and over time has access to increasingly superior technology. Nevertheless, this sector still grows more slowly than the more capital-intensive sector, and the non-balanced nature of the growth process remains. Therefore, endogenous 
growth does not restore balance between the two sectors as long as capital intensity (factor proportion) differences between the two sectors remain.

Unfortunately, we do not have an equivalent of Theorem 4 for the endogenous growth economy. The reason is that there are two more endogenous state variables and two more control variables now, so the dynamical system includes $c, \chi, \kappa, M_{1}, M_{2}, X_{1}$ and $X_{2}$ (or their stationary transformations). Given the size of this system, we are unable to prove local (saddle-path) stability. Nevertheless, Lemmas 3 and 4 imply that the system cannot lead to an explosive path, thus it has two tend to a constant growth path or settle into a limit cycle.

\section{Conclusions}

This paper shows how differences in factor proportions across sectors combined with capital deepening lead to a non-balanced pattern of economic growth. We first illustrated this point using a general two-sector growth model, and then characterized the equilibrium fully using a class of economies with constant elasticity of substitution between sectors and Cobb-Douglas production technologies. This class of economies shows how the pattern of equilibrium may be consistent with both non-balanced sectoral growth and the aggregate Kaldor facts, in particular, approximately constant share of capital in national income and interest rates. We also constructed and analyzed a model with endogenous technology featuring non-balanced economic growth.

The main contribution of the paper is theoretical, demonstrating that the interaction between capital deepening and factor proportion differences across sectors will lead to nonbalanced growth, while still remaining consistent with the aggregate Kaldor facts. We also presented a simple calibration of our baseline model, which showed that the equilibrium path may indeed exhibit sectoral employment and output shares changing significantly, while the aggregate capital share and the interest rate remain approximately constant. Moreover, the magnitudes implied by this simple calibration are comparable to, though somewhat smaller than, the sectoral changes observed in the the postwar US data. A full investigation of whether the mechanism suggested in this paper is first-order in practice is an empirical question left

for future research. It would be particularly useful to combine the mechanism proposed in this paper with non-homothetic preferences and estimate a structural version of the model with multiple sectors using US or OECD data. 


\section{Appendix A: Proof from Section 5}

\subsection{Proof of Lemma 3}

We will prove this lemma in four steps.

Step 1: $m_{1}^{*}=m_{2}^{*}=0$ imply $g_{2}^{*}=g_{1}^{*}=n$.

Step 2: $\limsup _{t \rightarrow \infty}\left(V_{1}-M_{1}^{\varphi} / b_{1}\right) \geq 0$ or $\limsup _{t \rightarrow \infty}\left(V_{2}-M_{2}^{\varphi} / b_{2}\right) \geq 0$.

Step 3: $\limsup _{t \rightarrow \infty}\left(V_{1}-M_{1}^{\varphi} / b_{1}\right) \geq 0$ and $\limsup _{t \rightarrow \infty}\left(V_{2}-M_{2}^{\varphi} / b_{2}\right) \geq 0$.

Step 4: $\lim _{t \rightarrow \infty}\left(V_{1}-M_{1}^{\varphi} / b_{1}\right)=0$ and $\lim _{t \rightarrow \infty}\left(V_{2}-M_{2}^{\varphi} / b_{2}\right)=0$.

Proof of Step 1: We first prove that $m_{1}^{*}=m_{2}^{*}=0$ imply $g_{2}^{*}=g_{1}^{*}=n$. To see this, combine equations (31) and (32) to obtain

$$
\left(\frac{Y_{2}}{Y_{1}}\right)^{\frac{1-\varepsilon}{\varepsilon}}=\left(\frac{1-\gamma}{\gamma}\right)\left(\frac{1-\alpha_{2}}{1-\alpha_{1}}\right) \frac{K_{1}}{K_{2}}
$$

Differentiating gives

$$
\frac{1-\varepsilon}{\varepsilon}\left(\alpha_{2} n_{2}-\alpha_{1} n_{1}\right)+\frac{1-\varepsilon}{\varepsilon}\left(1-\alpha_{2}\right) z_{2}-\frac{1-\varepsilon}{\varepsilon}\left(1-\alpha_{1}\right) z_{1}=z_{1}-z_{2} .
$$

To derive a contradiction, suppose that $g_{2}^{*}>g_{1}^{*}$. Differentiating (27), we obtain

$$
g_{1}=\alpha_{1} n_{1}+\left(1-\alpha_{1}\right) z_{1}+\frac{1}{\nu-1} m_{1} \text { and } g_{2}=\alpha_{2} n_{2}+\left(1-\alpha_{2}\right) z_{2}+\frac{1}{\nu-1} m_{2} .
$$

Then, from Lemma $1 n_{2}^{*}<n_{1}^{*}$ and $z_{2}^{*}<z_{1}^{*}$ and from Lemma $2, g^{*}=g_{1}^{*}$. Since $\lim _{t \rightarrow \infty} \dot{r}=0$, equation (46) and the fact that $g^{*}=g_{1}^{*}$ imply that $g_{1}^{*}=z_{1}^{*}$. This combined with equation (77) and $m_{1}^{*}=0$ implies that $z_{1}^{*}=n_{1}^{*}=n$, which together with (76) implies

$$
0>\frac{1-\varepsilon}{\varepsilon}\left(1-\alpha_{2}\right)\left(z_{2}^{*}-z_{1}^{*}\right)>z_{1}^{*}-z_{2}^{*},
$$

yielding a contradiction. The argument for the case in which $g_{2}^{*}<g_{1}^{*}$ is analogous. Since $g_{2}^{*}=g_{1}^{*}=g^{*}$ and $m_{1}^{*}=m_{2}^{*}=0$, it must also be the case that $g_{2}^{*}=g_{1}^{*}=n$, completing the proof of step 1 .

Proof of Step 2: First note that $\limsup _{t \rightarrow \infty}\left(V_{1}-M_{1}^{\varphi} / b_{1}\right)<0$ and $\lim \sup _{t \rightarrow \infty}\left(V_{2}-M_{2}^{\varphi} / b_{2}\right)<0$ imply that the free entry conditions, (68), are asymptotically slack, so $m_{1}^{*}$ and $m_{2}^{*}$ exist, and $\lim _{t \rightarrow \infty} m_{1}(t)=m_{1}^{*}=0$ and $\lim _{t \rightarrow \infty} m_{2}(t)=m_{2}^{*}=0$ (since they cannot be negative). In particular, note that if $\limsup _{t \rightarrow \infty}\left(V_{1}-M_{1}^{\varphi} / b_{1}\right)<0$, this implies that there exists no "infinitely-recurring" interval in the limit where the free entry condition holds for sector 1.

Now to derive a contradiction, suppose that $m_{2}^{*}=m_{1}^{*}=0$, which, as shown above, implies $g_{1}^{*}=$ $g_{2}^{*}=g^{*}=n>0$. Then, differentiating the second equation in (65), we obtain:

$$
\lim _{t \rightarrow \infty} \frac{\dot{\pi}_{2}}{\pi_{2}}=g^{*}>0
$$

Combining this with $\lim _{t \rightarrow \infty} \dot{r}=0$ and the value function in (62) yields: $\lim _{t \rightarrow \infty} V_{2}=\infty$. Since $m_{2}^{*}=0$ by hypothesis, $M_{2}^{-\varphi}$ is constant, and we have $\lim _{t \rightarrow \infty} V_{2}=\infty>\lim _{t \rightarrow \infty} M_{2}^{\varphi} / b_{2}$, violating the free entry condition (68). This proves that $m_{1}^{*}$ and $m_{2}^{*}$ cannot both equal to 0 , and thus $\lim \sup _{t \rightarrow \infty}\left(V_{2}-M_{2}^{\varphi} / b_{2}\right)<0$ and $\limsup _{t \rightarrow \infty}\left(V_{1}-M_{1}^{\varphi} / b_{1}\right)<0$ is not possible.

Proof of Step 3: We next prove that $\lim _{\sup _{t \rightarrow \infty}}\left(V_{1}-M_{1}^{\varphi} / b_{1}\right) \geq 0$, $\lim \sup _{t \rightarrow \infty}\left(V_{2}-M_{2}^{\varphi} / b_{2}\right) \geq 0, \limsup _{t \rightarrow \infty} m_{1}>0$ and $\lim \sup _{t \rightarrow \infty} m_{2}>0$. 
Suppose, to derive a contradiction, $\lim \sup _{t \rightarrow \infty} m_{2}=m_{2}^{*}=0$ and

$\lim \sup _{t \rightarrow \infty}\left(V_{2}-M_{2}^{\varphi} / b_{2}\right)<0$ (the other case is proved analogously). Since, as shown above, $m_{1}^{*}=$ $m_{2}^{*}=0$ is not possible, we must have $\lim \sup _{t \rightarrow \infty}\left(V_{1}-M_{1}^{\varphi} / b_{1}\right) \geq 0$ and $\lim \sup _{t \rightarrow \infty} m_{1}>0$.

We start by noting from (65), that asymptotically

$$
\lim _{t \rightarrow \infty} \frac{\dot{\pi}_{2}}{\pi_{2}}=\frac{1}{\varepsilon}\left(g-g_{2}\right)+g_{2}
$$

because $m_{2}^{*}=0$. Since

$$
\lim _{t \rightarrow \infty} \frac{\dot{r}}{r}=\frac{1}{\varepsilon}\left(g-g_{2}\right)+g_{2}-z_{2}=0
$$

from (32), $z_{2}>0$ implies that $\lim _{t \rightarrow \infty} \dot{\pi}_{2} / \pi_{2}>0$. But by the same argument as in Step 2, we have $\lim _{t \rightarrow \infty} V_{2}=\infty>\lim _{t \rightarrow \infty} M_{2}^{\varphi} / b_{2}$, violating the free entry condition (68). We therefore only have to show that $z_{2}>0$ (which would naturally imply that $\lim _{\sup _{t \rightarrow \infty}} z_{2}>0$ ). Suppose, to obtain a contradiction, that $z_{2}^{*}=0$. Using (45) and (46) from the proof of Lemma 1 , we have $z_{2}-n_{2}=z_{1}-n_{1}$, which implies that $n_{2}^{*}=0$ (recall that either $n_{1} \geq n$ or $n_{2} \geq n$, and since $\lim _{t \rightarrow \infty} g \geq n$, either $z_{1} \geq n$ or $\left.z_{2} \geq n\right)$. But then with $n_{2}^{*}=z_{2}^{*}=m_{2}^{*}=0$, we have $g_{2}^{*}=0<g_{1}^{*}$, which contradicts $n_{2}^{*}<n_{1}^{*}=n$ from Lemma 1. A similar argument for the other sector completes the proof that $\lim \sup _{t \rightarrow \infty}\left(V_{1}-M_{1}^{\varphi} / b_{1}\right) \geq 0$ and $\lim \sup _{t \rightarrow \infty}\left(V_{2}-M_{2}^{\varphi} / b_{2}\right) \geq 0$.

Proof of Step 4. From the free entry conditions in (68), we have that $V_{1}-M_{1}^{\varphi} / b_{1} \leq 0$ and $V_{2}-M_{2}^{\varphi} / b_{2} \leq 0$, thus $\lim \sup _{t \rightarrow \infty}\left(V_{1}-M_{1}^{\varphi} / b_{1}\right) \leq 0$ and $\limsup _{t \rightarrow \infty}\left(V_{2}-M_{2}^{\varphi} / b_{2}\right) \leq 0$. Combined with Step 3, this implies $\lim \sup _{t \rightarrow \infty}\left(V_{1}-M_{1}^{\varphi} / b_{1}\right)=0$ and $\lim \sup _{t \rightarrow \infty}\left(V_{2}-M_{2}^{\varphi} / b_{2}\right)=0$. Hence, we only have to prove that $\liminf _{t \rightarrow \infty}\left(V_{1}-M_{1}^{\varphi} / b_{1}\right) \geq 0$ and $\liminf _{t \rightarrow \infty}\left(V_{2}-M_{2}^{\varphi} / b_{2}\right) \geq 0$. We prove the first inequality (the proof of the second is similar).

Suppose, to derive a contradiction, that $\liminf _{t \rightarrow \infty}\left(V_{1}-M_{1}^{\varphi} / b_{1}\right)<0$. This implies that there exists a (recurring) interval $\left(t_{0}^{\prime}, t_{2}^{\prime}\right)$ such that $V_{1}(t)-M_{1}^{\varphi}(t) / b_{1}<0$ for all $t \in\left(t_{0}^{\prime}, t_{2}^{\prime}\right)$. Suppose that $\left(t_{0}^{\prime}, t_{2}^{\prime}\right)$ is unbounded; this would imply that $\limsup _{t \rightarrow \infty} m_{1}=m_{1}^{*}=0$, yielding a contradiction with Step 2. Thus $\left(t_{0}^{\prime}, t_{2}^{\prime}\right)$ must be bounded, so there exists $\left(t_{0}, t_{2}\right) \supset\left(t_{0}^{\prime}, t_{2}^{\prime}\right)$ such that for $t \in\left(t_{0}, t_{2}\right) \backslash\left(t_{0}^{\prime}, t_{2}^{\prime}\right)$, we have $V_{1}(t)-M_{1}^{\varphi}(t) / b_{1}=0$. Moreover, since $\limsup _{t \rightarrow \infty} m_{1}>0$, there also exists an interval $\left(t_{0}^{\prime \prime}, t_{2}^{\prime \prime}\right) \supset\left(t_{0}, t_{2}\right)$ such that for all $t \in\left(t_{0}, t_{2}\right) \backslash\left(t_{0}^{\prime \prime}, t_{2}^{\prime \prime}\right), m_{1}>0$.

Next, since $m_{1}=0$ for all $t \in\left(t_{0}^{\prime}, t_{2}^{\prime}\right)$, we also have $M_{1}\left(t_{0}^{\prime}\right)=M_{1}\left(t_{2}^{\prime}\right)$. This implies

$$
V_{1}\left(t_{2}^{\prime}\right)=\frac{M_{1}^{\varphi}\left(t_{0}^{\prime}\right)}{b_{1}}=V_{1}\left(t_{0}^{\prime}\right) .
$$

Figure A1 shows this diagrammatically.

Let us rewrite (62) in the Bellman equation form

$$
\frac{\dot{V}_{1}(t)}{r}=V_{1}(t)-\frac{\pi_{1}(t)}{r} \quad \forall t
$$

Equation (81) also shows that $\dot{V}_{1}(t)$ is well-defined, so $V_{1}(t)$ is continuously differetiable in $t$. Equation (80) and the fact that $V_{1}(t)-M_{1}^{\varphi}\left(t_{0}^{\prime}\right) / b_{1}<0$ for all $t \in\left(t_{0}^{\prime}, t_{2}^{\prime}\right)$ imply that $V_{1}(t)$ reaches a minimum over $\left(t_{0}^{\prime}, t_{2}^{\prime}\right)$ with $\dot{V}_{1}(t)=0$. Let $t_{1}^{\prime}<t_{2}^{\prime}$ be such that $V_{1}\left(t_{1}^{\prime}\right)$ is the first local minimum after $t_{0}^{\prime}$, which naturally satisfies $V_{1}\left(t_{1}^{\prime}\right)<V_{1}\left(t_{0}^{\prime}\right)$. Moreover, using (65) and (67), we have that for all $t$

$$
\frac{\pi_{1}(t)}{r^{*}}=\frac{1}{(\nu-1)\left(1-\alpha_{1}\right)} \frac{K_{1}(t)}{M_{1}(t)},
$$

where $r^{*}=\lim _{t \rightarrow \infty} r(t)$ is the asymptotic equilibrium interest rate, which exists by hypothesis that $\lim _{t \rightarrow \infty} \dot{r}(t)=0$. Also, using the fact that $\lim _{t \rightarrow \infty} \dot{r}(t)=0$ and the interest rate equation, (67), we 
obtain that since $m_{1}=0$ and $n>0, K_{1}\left(t_{1}^{\prime}\right)>K_{1}\left(t_{0}^{\prime}\right)$. In addition, since $M_{1}\left(t_{1}^{\prime}\right)=M_{1}\left(t_{0}^{\prime}\right)$, we can use (81) to write

$$
\frac{\dot{V}_{1}\left(t_{1}^{\prime}\right)}{r^{*}}=V_{1}\left(t_{1}^{\prime}\right)-\frac{1}{(\nu-1)\left(1-\alpha_{1}\right)} \frac{K_{1}\left(t_{1}^{\prime}\right)}{M_{1}\left(t_{1}^{\prime}\right)}<V_{1}\left(t_{0}^{\prime}\right)-\frac{1}{(\nu-1)\left(1-\alpha_{1}\right)} \frac{K_{1}\left(t_{0}^{\prime}\right)}{M_{1}\left(t_{0}^{\prime}\right)}=\frac{\dot{V}_{1}\left(t_{0}^{\prime}\right)}{r^{*}}<0
$$

which contradicts the fact that $V_{1}\left(t_{1}^{\prime}\right)$ is a local minimum, completing the proof of the lemma.

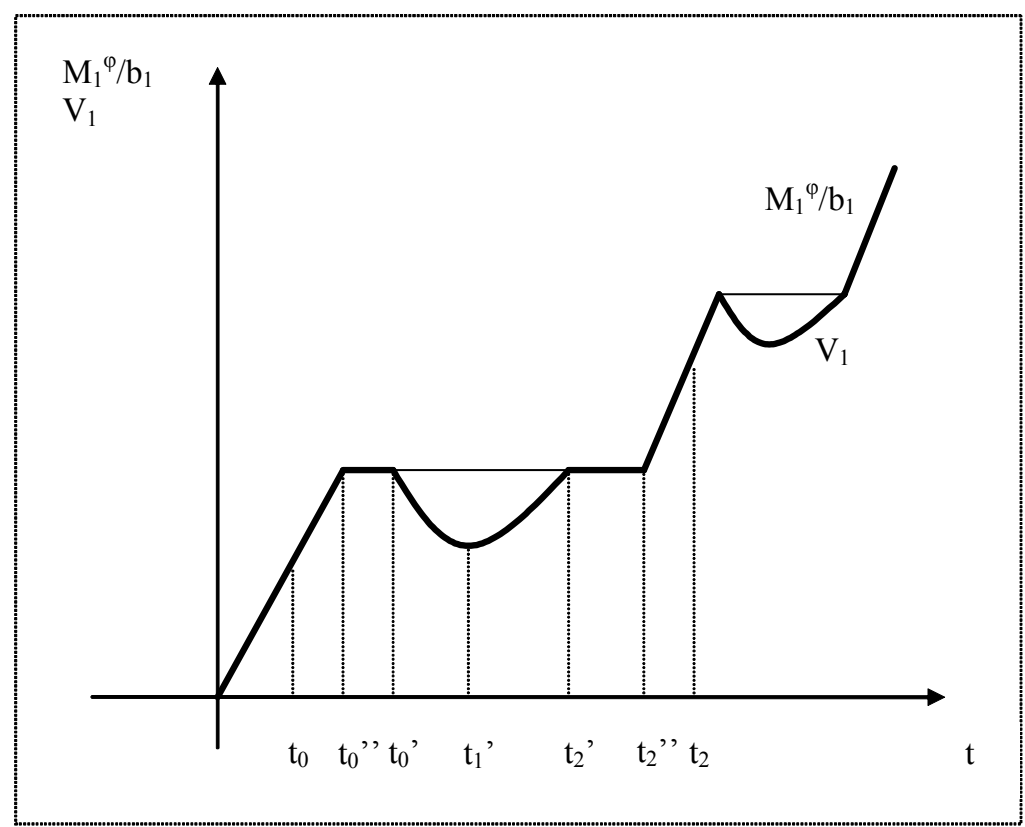

Figure A1: The solid line represents $M_{1}^{\varphi} / b_{1}$ and the thick line represents $V_{1}$.

\subsection{Proof of Lemma 4 and The Converse Result}

Proof of Lemma 4: First, recall that $C \leq Y$, (14). Hence it is enough to prove that $\lim _{t \rightarrow \infty} g=\infty$ will violate the resource constraint. We will prove this separately in two cases, when $g_{2}^{*} \geq g_{1}^{*}$ and when $g_{2}^{*}<g_{1}^{*}$

Suppose $g_{2}^{*} \geq g_{1}^{*}$ and $g^{*}=\infty$. Recall that Lemmas 1 and 2 from Section 3 still apply. In particular, Lemma 2 implies $g_{1}^{*}=g^{*}=\infty$, and equation (46) together with (77) yields

$$
g=n-\left(\frac{1-\alpha_{1}}{\alpha_{1}}\right) \frac{\dot{r}}{r}+\frac{1}{\alpha_{1}(\nu-1)} m_{1} .
$$

Given $n<\infty$ and $\lim _{t \rightarrow \infty} \dot{r} / r \geq 0$, it must be that asymptotically

$$
g^{*}=\frac{1}{\alpha_{1}(\nu-1)} m_{1}^{*}
$$

Combining the technology possibility frontier (61) and (82) we have

$$
\lim _{t \rightarrow \infty} \frac{\dot{X}_{1}}{X_{1}}=\lim _{t \rightarrow \infty} \frac{\dot{m}_{1}}{m_{1}}+\alpha_{1}(1+\varphi)(\nu-1) g^{*} .
$$


Then, the first inequality in Assumption A3, $\zeta>1 / \alpha_{1}$, implies

$$
\lim _{t \rightarrow \infty} \frac{\dot{X}_{1}}{X_{1}}>g^{*}
$$

which violates the resource constraint (69).

Next suppose that $g_{1}^{*}>g_{2}^{*}$ and $g^{*}=\infty$. Then, following the same steps above, Lemma 2 implies $g_{2}^{*}=g^{*}=\infty$, and equation (46) together with (45) and (77) yields

$$
\left(\frac{1-\alpha_{1}(1-\varepsilon)}{\varepsilon}\right) g_{1}=\alpha_{1} n_{1}+\left(\frac{1-\alpha_{1}}{\varepsilon}\right) g-\left(1-\alpha_{1}\right) \frac{\dot{r}}{r}+\left(\frac{1}{\nu-1}\right) m_{1} .
$$

Since $g^{*}=\infty$, a fortiori $g_{1}^{*}=\infty$, and, given $n<\infty$ and $\lim _{t \rightarrow \infty} \dot{r} / r \geq 0$, we have that asymptotically

$$
m_{1}^{*}=(\nu-1)\left[\left(\frac{1-\alpha_{1}(1-\varepsilon)}{\varepsilon}\right) g_{1}^{*}-\left(\frac{1-\alpha_{1}}{\varepsilon}\right) g^{*}\right] \text {. }
$$

Once again the innovation possibilities frontier (61) implies

$$
\lim _{t \rightarrow \infty} \frac{\dot{X}_{1}}{X_{1}}=\lim _{t \rightarrow \infty} \frac{\dot{m}_{1}}{m_{1}}+\alpha_{1}(1-\varphi) m_{1}^{*}
$$

Then equation (83) together with the first inequality in Assumption A3, $\zeta>1 / \alpha_{1}$, implies

$$
\lim _{t \rightarrow \infty} \frac{\dot{X}_{1}}{X_{1}}>\alpha_{1}(1-\varphi)(\nu-1) g^{*}>g^{*},
$$

which violates the resource constraint (69), completing the proof that when Assumption A3 holds, any quasi-equilibrium with more than exponential growth violates the resource constraint.

For completeness, we also prove the converse of Lemma 4, which shows that the use of the first inequality in Assumption A3, $\zeta>1 / \alpha_{1}$, in this lemma is "tight" in the sense that, if it were relaxed, the opposite result would obtain.

Lemma 4': Suppose A1 holds, but $\zeta \equiv(\nu-1)(1-\varphi) \leq 1 / \alpha_{1}$, then there exists quasi-equilibria with $\lim _{t \rightarrow \infty} g=\infty$.

Proof. This lemma will be proved by showing that in this case

$$
\begin{gathered}
g_{2}^{*}=g_{1}^{*}=g^{*}=\infty \quad \text { and } \quad z_{2}^{*}=z_{1}^{*}=z^{*} \\
z^{*}=g^{*}-\frac{\dot{r}}{r} \\
\frac{\dot{m}_{1}}{m_{1}}=\frac{\dot{m}_{2}}{m_{2}}=\left[1-\alpha_{1} \zeta\right] g
\end{gathered}
$$

is a quasi-equilibrium.

Combining equation (46) from Section 3 with (84), we obtain

$$
z^{*}=g^{*}-\lim _{t \rightarrow \infty} \frac{\dot{r}}{r}
$$

which is exactly condition (85). By substituting into (77), we obtain

$$
g^{*}=n_{1}-\left(\frac{1-\alpha_{1}}{\alpha_{1}}\right) \lim _{t \rightarrow \infty} \frac{\dot{r}}{r}+\frac{1}{\alpha_{1}(\nu-1)} m_{1}^{*}
$$




$$
g^{*}=n_{2}-\left(\frac{1-\alpha_{2}}{\alpha_{2}}\right) \lim _{t \rightarrow \infty} \frac{\dot{r}}{r}+\frac{1}{\alpha_{2}(\nu-1)} m_{2}^{*}
$$

which gives $g^{*}=\frac{1}{\alpha_{1}(\nu-1)} m_{1}^{*}$ and $g^{*}=\frac{1}{\alpha_{2}(\nu-1)} m_{2}^{*}$, and hence

$$
m_{1}^{*}=\frac{\alpha_{1}}{\alpha_{2}} m_{2}^{*}
$$

Differentiating this condition gives the first equality of equation (86).

Next, from the technology possibilities frontiers, (61), feasibility requires

$$
\begin{aligned}
& \lim _{t \rightarrow \infty} \frac{\dot{X}_{1}}{X_{1}}=\frac{\dot{m}_{1}^{*}}{m_{1}^{*}}+\alpha_{1} \zeta g^{*} \leq g^{*} \\
& \lim _{t \rightarrow \infty} \frac{\dot{X}_{2}}{X_{2}}=\frac{\dot{m}_{2}^{*}}{m_{2}^{*}}+\alpha_{2} \zeta g^{*} \leq g^{*}
\end{aligned}
$$

and both these conditions are satisfied given the second equality of equation (86) and $\alpha_{1}>\alpha_{2}$.

\subsection{Proof of Theorem 5}

We prove this proposition in two steps.

Step 1: Provided that $g_{2}^{*} \geq g_{1}^{*}>0$, then there exists a unique CGP defined by equations (71), (72), (73), (74) and (75), satisfying $g_{2}^{*}>g_{1}^{*}>0$.

Step 2: All CGP must satisfy $g_{2}^{*} \geq g_{1}^{*}>0$.

Proof of Step 1. Lemma 3 establishes that as $t \rightarrow \infty$ the free-entry conditions (68) must asymptotically hold as equality. Combining (68) as equality with (81) (and the equivalent for sector 2), we obtain the following conditions that must hold as $t \rightarrow \infty$ :

$$
\frac{\frac{\gamma}{\nu}\left(\frac{Y_{1}}{Y}\right)^{-\frac{1}{\varepsilon}} Y_{1}}{r-\varphi m_{1}}=\frac{M_{1}^{1+\varphi}}{b_{1}} \text { and } \frac{\frac{\gamma}{\nu}\left(\frac{Y_{2}}{Y}\right)^{-\frac{1}{\varepsilon}} Y_{2}}{r-\varphi m_{2}}=\frac{M_{2}^{1+\varphi}}{b_{2}}
$$

Differentiating (87) yields:

$$
g_{1}-\frac{1}{\varepsilon}\left(g_{1}-g\right)-(1+\varphi) m_{1}=0 \text { and } g_{2}-\frac{1}{\varepsilon}\left(g_{2}-g\right)-(1+\varphi) m_{2}=0 .
$$

Then $g_{2}^{*}>g_{1}^{*}>0$ and Lemma 2 imply that we must also have $g^{*}=g_{1}^{*}$. This condition together our system of equations, (45), (46), (77), (88), solves uniquely for $n_{1}^{*}, n_{2}^{*}, z_{1}^{*}, z_{2}^{*}, m_{1}^{*}, m_{2}^{*}, g_{1}^{*}$ and $g_{2}^{*}$ (and $\left.\kappa^{*}=\lambda^{*}=1\right)$ as given in equations (71), (72), (73), (74) and (75). Note that this solution is consistent with $g_{2}^{*}>g_{1}^{*}>0$, since Assumptions A1 and A3 immediately imply that $g_{2}^{*}>g_{1}^{*}$ and $g_{1}^{*}>0$ (which is also consistent with Lemma 3). Finally, $C \leq Y$, (14) and (44) imply that the consumption growth rate, $g_{C}^{*}$, is equal to the growth rate of output, $g^{*}$.

Finally, we can verify that an equilibrium with $\kappa^{*}, \lambda^{*}, n_{1}^{*}, n_{2}^{*}, z_{1}^{*}, z_{2}^{*}, m_{1}^{*}, m_{2}^{*}, g_{1}^{*}$ and $g_{2}^{*}$ satisfies the transversality condition (44) with a similar argument to the one spelled in the first step of the proof of Theorem 3 (since Assumption A3 ensures that $g^{*}<r^{*}$ ).

Proof of Step 2. The proof that along all CGPs $g_{2}^{*}>g_{1}^{*}>0$ must be true, is analogous to the one of the second step in the proof of Theorem 3. 


\section{Appendix B: National Income Product Accounts Data}

All the data used in the paper refer to US data and are from National Income and Product Accounts (NIPA). Sectoral data are from the Gross Domestic Product by Industry Data of the NIPA. Sectors are classified according to the North American Industrial Classification System (NAICS). Throughout, we use the 22-industry level of detail data, which corresponds to two-digit industries. This level of aggregation enables us to extend the sample back to 1948. We exclude the Government and Private household sectors as well as Agriculture, forestry, fishing, and hunting and Real estate and rental. Agriculture is excluded since our focus is on services and manufacturing and the difference between more and less capital-intensive sectors. Real estate is excluded since it has a very high capital share due to the value of assets in this sector, which does not reflect the share of capital in the production function of the sector. The nominal value added and employment figures for the aggregate economy we use throughout exclude the sectors.

In Figure 1, "Services" are: Wholesale and Retail trade; Transportation and Warehousing; Information; Finance and Insurance; Professional, scientific, and technical services; Management of companies and enterprises; Administrative and waste management services; Educational services; Health care and social assistance; Arts, Entertainment, and Recreation; Accomodation and food services; Other services, except government. Manufacturing includes: Mining; Utilities; Construction; Durable and Non-durable goods.

Employment in Figure 1 and throughout is total full-time equivalent employment (FTE), in thousands, in the indicated industries. Real GDP is equal to expenditure-weighted sum of real value added. More specifically, let $\mathcal{S}$ denote a subset of the two-digit sectors. Then real GDP in this subset of sectors at time $t$ is calculated as:

$$
\text { real } \operatorname{GDP}_{t}^{\mathcal{S}}=\frac{\sum_{j \in S} E_{j, t} \cdot \mathrm{RVA}_{j, t}}{\sum_{j \in S} E_{j, t}},
$$

where $\mathrm{RVA}_{j, t}$ is real value added in sector $j$ at time $t$ in 2000 dollars, obtained by deflating nominal value added (VA) by NIPA's chain-weighted price index (VAPI, and the corresponding chain-weighted quantity index, VAQI), and $E_{j, t}$ is expenditure on sector $j$ at time $t$, approximated by nominal value added.

The share of capital in national income is computed as value added minus total compensation to employees over value added, i.e.,

$$
\text { capital } \operatorname{share}_{t}=\frac{E_{t}-W_{t}}{E_{t}},
$$

where $E_{t}$ is total nominal value added (VA) at time $t$ and $W_{t}$ is total nominal compensation to employees (COMP) at time $t$.

The value of initial capital stock is the initial value of private fixed assets in current dollars.

In Figure 2 and in Section 4, sectors are classified according to their capital intensity. Capital intensity in each sector is calculated as:

$$
\text { capital share }_{j, t}=\frac{E_{j, t}-W_{j, t}}{E_{j, t}},
$$

where $E_{j, t}$ is nominal value added in sector $j$ at time $t$ and $W_{j, t}$ is nominal compensation to employees in sector $j$ at time $t$. Because of the change in the classification of industries before and after 1987, we compute the capital share of each industry as the average between 1987 and 2004, which enables us to use the consistent NAICS classification for the compensation series (before 1987 this variable is only available with the SIC classification). Sectors are then ranked according to their average capital intensity as shown in Table B1 below, and the capital share of 0.35 is chosen as the cutoff level for separating industries into high and low capital-intensity sectors, which creates two groups of industries, each with approximately $50 \%$ of employment. According to this ranking, low capital-intensity sectors are: Construction; Durable goods; Transportation and Warehousing; Professional, scientific, and technical services; Management 
of companies and enterprises; Administrative and waste management services; Educational services; Health care and social assistance; Other services, except government. High capital-intensive sectors are: Mining; Utilities; Non-durable goods; Wholesale; Retail trade; Information; Finance and Insurance; Arts, Entertainment, and Recreation; Accomodation and food services. These industries, their average capital shares and their allocations to sectors 1 and 2 are shown in Table B1.

Table B1: Industry Capital Shares

\begin{tabular}{|l|c|c|}
\hline \hline INDUSTRY & SECTOR & CAPITAL SHARE \\
\hline \hline Educational services & 1 & 0.09 \\
Management of companies and enterprises & 1 & 0.19 \\
Health care and social assistance & 1 & 0.22 \\
Durable goods & 1 & 0.27 \\
Administrative and waste management services & 1 & 0.28 \\
Construction & 1 & 0.32 \\
Other services, except government & 1 & 0.34 \\
Professional, scientific, and technical services & 1 & 0.34 \\
Transportation and warehousing & 1 & 0.34 \\
\hline Accomodation and food services & 2 & 0.35 \\
Retail trade & 2 & 0.42 \\
Arts, entertainment, and recreation & 2 & 0.43 \\
Finance and insurance & 2 & 0.46 \\
Wholesale trade & 2 & 0.46 \\
Nondurable goods & 2 & 0.46 \\
Information & 2 & 0.53 \\
Mining & 2 & 0.62 \\
Utilities & 2 & 0.77 \\
\hline \hline
\end{tabular}

Note: Capital shares refer to the average capital share between 1987 and 2004 for each industry computed according to equation (90).

Finally, note that in both Figures 1 and 2, there is a slight break in the employment series in 1997 which is due to a change in the industry classification system used in the NIPA. The employment series in NIPA are classified with the SIC system between 1948 and 1997 and using the the NAICS from 1998 onwards. We match the sectors in the two classifications using the correspondence tables constructed by the US Census Bureau. We also use the same tables to extend total compensations to employees back to 1948 in order to compute the initial aggregate capital share of the economy. 


\section{References}

[1] Acemoglu, Daron, "Directed Technical Change" Review of Economic Studies, LXIX (2002), 781-810.

[2] Acemoglu, Daron, "Labor- and Capital-Augmenting Technical Change" Journal of European Economic Association, I (2003), 1-37.

[3] Aghion, Philippe and Peter Howitt, "A Model of Growth Through Creative Destruction" Econometrica, LX (1992), 323-351.

[4] Aghion, Philippe and Peter Howitt, Endogenous Growth Theory, Cambridge, MA, MIT Press, 1998.

[5] Antras, Pol, "Is the U.S. Aggregate Production Function Cobb-Douglas? New Estimates of the Elasticity of Substitution" MIT mimeo (2001).

[6] Barro, Robert and Xavier Sala-i-Martin. Economic Growth, McGraw Hill, New York (2004, 2nd edition).

[7] Baumol, William J., "Macroeconomics of Unbalanced Growth: The Anatomy of Urban Crisis", American Economic Review, LVII (1967), 415-426.

[8] Berndt, Ernst, "Reconciling Alternative Estimates of the Elasticity of Substitution," Review of Economics and Statistics, LVIII (1976), 59-68.

[9] Bewley, Truman, "An Integration of Equilibrium Theory and Turnpike Theory" Journal of Mathematical Economics, X (1982), 233-267.

[10] Buera, Francisco and Joseph Kaboski "The Rise of the Service Economy" Northwestern mimeo, 2006.

[11] Caselli, Francesco and John Coleman, "The U. S. Structural Transformation and Regional Convergence: a Reinterpretation" Journal of Political Economy CIX (2001) 584-617.

[12] Chenery, Hollis, "Patterns of Industrial Growth", American Economic Review, V (1960), 624-654. 
[13] Chirinko, Robert S. "Business Fixed Investment: a Critical Survey of Modeling Strategies, Empirical Results and Policy Implications" Journal of Economic Literature, XXXI, (1993), 1875-1911.

[14] Chirinko, Robert S., Steven M. Fazzari and Andrew P. Mayer, "How Responsive Is Business Capital Formation to Its User Cost?" Journal of Public Economics, LXXV, (1999), $53-80$.

[15] David, Paul, and Th. Van de Klundert, "Biased Efficiency Growth and Capital-Labor Substitution in the U.S., 1899-1960," American Economic Review, LV, (1965), 357-393.

[16] Denison, Edward F, “Accounting for United States Economic Growth, 1929-1969” Washington, DC: Brookings Institution (1974).

[17] Echevarria, Cristina, "Changes in Sectoral Composition Associated with Economic Growth", International Economic Review, XXXVIII (1997), 431-452.

[18] Foellmi, Reto and Josef Zweimuller "Structural Change and the Kaldor Facts of Economic Growth" CEPR Discussion Paper, No. 3300, 2002.

[19] Gollin, Douglas, Stephen Parente and Richard Rogerson "The Role of Agriculture in Development" American Economic Review Papers and Proceedings XCII (2002) 160-164.

[20] Griffin, James M. and Paul R. Gregory, "An Intercountry Translog Model of Energy Substitution Responses", American Economic Review, LXVI (1976), 845-857.

[21] Grossman, Gene and Elhanan Helpman, "Quality Ladders in the Theory of Growth" Review of Economic Studies, LIIX (1991a), 43-61.

[22] Grossman, Gene and Elhanan Helpman, Innovation and Growth in the Global Economy, Cambridge, MA, MIT Press, 1991b.

[23] Hall, Robert E. and Charles I. Jones "The Value of Life and the Rise in Health Spending" forthcoming Quarterly Journal of Economics, (2006).

[24] Hamermesh, David S., Labor Demand, Princeton University Press, Princeton 1993.

[25] Homer, Sydney and Richard Sylla A History of Interest Rates, Rutgers University Press, New Brunswick, 1991. 
[26] Howitt, Peter, "Steady Endogenous Growth with Population and R\&D Inputs Growing" Journal of Political Economics, CVII (1999), 715-730.

[27] Jensen, Martin K. "Balanced Growth, Dynamic Stability and the Turnpike" University of Copenhagen, Ph.D. dissertation, 2002.

[28] Jones, Charles I., "R\&D-Based Models of Economic Growth", Journal of Political Economics, CIII (1995), 759-784.

[29] Jones, Charles I., "The Shape of Production Functions and the Direction of Technical Change", U.C. Berkeley mimeo (2004).

[30] Judd, Kenneth, Numerical Methods in Economics, MIT Press, Cambridge, 1998.

[31] Kaldor, Nicholas , "Capital Accumulation and Economic Growth", in Friedrich A. Lutz and Douglas C. Hague, eds., Proceedings of a Conference Held by the International Economics Association, London, Macmillan.(1963)

[32] Kongsamut, Piyabha , Sergio Rebelo and Danyang Xie, "Beyond Balanced Growth", Review of Economic Studies, LXVIII (2001), 869-882.

[33] Kortum, Samuel, "Research, Patenting and Technological Change" Econometrica, LXV (1997), 1389-1431.

[34] Krusell, Per; Lee Ohanian and Victor Rios-Rull and Giovanni Violante, "Capital Skill Complementary and Inequality", Econometrica, LXIIX (2000), 1029-1053.

[35] Kuznets, Simon, "Quantitative Aspects of the Economic Growth of Nations: II, Industrial Distribution of National Product and Labour Forcce", Economic Development and Cultural Change, V Supplement (1957).

[36] Kuznets, Simon, "Modern Economic Growth: Findings and Reflections", American Economic Review, LXIII (1973), 829-846.

[37] Laitner, John, "Structural Change and Economic Growth", Review of Economic Studies, LXVII (2000), 545-561.

[38] Lucas, Robert E., "On the mechanics of economic development." Journal of Monetary Economics, XXII (1988), 3-42. 
[39] Matsuyama, Kiminori, "Agricultural Productivity, Comparative Advantage and Economic Growth" Journal of Economic Theory LVIII (1992), 317-334.

[40] Matsuyama, Kiminori, "The Rise of Mass Consumption Societies" Journal of Political Economy, CX (2002), 1093-1120.

[41] Matsuyama, Kiminori, "Structural Change" forthcoming New Pelgrave Dictionary of Economics (2005).

[42] Mairesse, Jacques, Bronwyn H. Hall and Benoit Mulkay, "Firm-Level Investment in France and the United States: An Exploration over What We Have Returned in Twenty Years" Annales d'Economie et Statistiques, LV (1999), 27-67.

[43] McKenzie, Lionel W., "Turnpikes: Richard Ely Lecture" American Economic Review, (1998).

[44] Murphy, Kevin, Andrei Shleifer and Robert Vishny, "Income Distribution, Market Size and Industrialization" Quarterly Journal of Economics CIV (1989), 537-564.

[45] Nadiri, M. I. "Some Approaches to Theory and Measurement of Total Factor Productivity: a Survey" Journal of Economic Literature, VIII, (1970), 1117-77.

[46] Nerlove, Mark, "Recent Empirical Studies of the CES and Related Production Functions" in M. Brown (editor) The Theory and Empirical Analysis of Production New York (1967).

[47] Ngai Rachel and Christopher Pissarides, "Structural Change in a Multi-Sector Model of Growth", London School of Economics, mimeo, 2006.

[48] Radner, Roy "Paths of Economic Growth That Are Optimal With Regard Only to Final States" Review of Economic Studies, XXVIII (1961), 98-104.

[49] Romer, Paul M., "Increasing Returns and Long-Run Growth" Journal of Political Economy, XCIV (1986), 1002-1037.

[50] Romer, Paul M., "Endogenous Technological Change" Journal of Political Economy, IIC (1990), S71-S102.

[51] Rybczynski, Tadeusz, "Factor Endowments and Relative Commodity Prices" Economica, XXII (1955), 336-341. 
[52] Scheinkman, José A. "On Optimal Steady States of n-Sector Growth Models When Utility Is Discounted" Journal of Economic Theory, XII (1976), 11-20.

[53] Segerstrom, Paul S., T. C. A. Anant, and Elias Dinopoulos, "A Schumpeterian Model of the Product Life Cycle" American Economic Review LXXX (1990), 1077-1092.

[54] Stokey, Nancy "Learning by Doing in the Introduction of New Goods" Journal of Political Economy, XCVI (1988), 701-717.

[55] Stokey, Nancy, "Human Capital, Product Quality and Growth" Quarterly Journal of Economics, CVI (1991), 587-616.

[56] Young, Alwyn "Invention and Bounded Learning by Doing" Journal of Political Economy CI (1993), 443-472.

[57] Young, Alwyn "Growth without Scale Effect" Journal of Political Economy, CVI (1998), 41-63.

[58] Zuleta, Hernando and Andrew Young "Labor's Shares-Aggregate and Industry: Accounting for Both in a Model with Induced Innovation" University of Mississippi, mimeo, 2006. 